\title{
A high order finite difference solver for massively parallel simulations of stably stratified turbulent channel flows
}

Ping $\mathrm{He}^{*}$

Department of Marine, Earth, and Atmospheric Sciences, North Carolina State University

\begin{abstract}
Investigation of stably stratified turbulent flows in the atmosphere is challenging owing to its nonstationarity and heterogeneity, especially in very stable conditions. In recent years, numerical simulation is becoming a powerful tool to complement the understanding of fundamental characteristics of strongly stratified flows. Owing to its highly accurate representation of small-scale turbulence structures, the direct numerical simulation (DNS) is shown to be the most effective numerical approach for strongly stratified flows. However, due to the limitation of the traditional parallel strategy, the existing DNS studies on stratified channel flows are limited to relatively low Reynolds numbers. In order to make the DNS studies more relevant to the atmospheric boundary layer flows, in this paper, a high-order finite-difference DNS solver is developed for simulating stably stratified turbulent channel flows at relatively high Reynolds numbers. Utilizing the advanced two dimensional domain decomposition technique, the DNS solver is shown to be competent for petascale simulations with excellent scalability tested up to $32768 \mathrm{CPU}$ cores. Using this newly developed solver, two stably stratified open-channel simulations are conducted with the highest Reynolds number being approximately $10^{5}$. The fundamental characteristics of strongly stratified turbulent flows, e.g., spatio-temporal variation of global intermittency, are compared with those previously reported at relatively low Reynolds numbers, and their similarities and discrepancies are also discussed. In addition, the DNS-based Monin-Obukhov similarity relationships are compared with those proposed based on observational data to evaluate their relevance to atmospheric flows. The source code and the simulation results are available at http://sites.google.com/site/scdnscode/
\end{abstract}

\footnotetext{
${ }^{*}$ Corresponding author

Email address: phe3@ncsu.edu (Ping He )
} 
Keywords: Direct numerical simulation, intermittent turbulence, petascale parallel simulation, scalability, stably stratified flows

\section{Introduction}

In the nocturnal atmospheric boundary layer (ABL), the surface is cooled and the turbulence is typically stably stratified. For weakly stable conditions, the turbulence structures are commonly stationary and horizontally homogeneous, and they are relatively well described

5 by the classic ABL theories, e.g., see the textbooks $[1,2,3,4]$. However, in very stable conditions, turbulence transport and mixing are significantly modulated by buoyancy, resulting in strongly heterogeneous and nonstationary turbulence activities, e.g., gravity waves, intermittent turbulence. Although numerous studies have been conducted to investigate the behaviors of the very stable ABL, its fundamental characteristics are still far from fully understood (please refer to Mahrt [5] for a review of current challenges in the very stable $A B L)$. From a practical standpoint, investigation of stably stratified flows is related to various industrial, environmental, and geophysical applications, e.g., wind energy estimation $[6,7]$, transport of pollutants in the $\mathrm{ABL}[8,9,10]$, mixing parameterization for numerical weather prediction models [11].

In recent years, numerical simulation is becoming a powerful tool to complement the understanding of the underlying dynamics and physics of the very stable ABL. The large-eddy simulation (LES) is one of the most efficient computational techniques for high Reynolds number turbulent flow simulations. However, its applicability for strongly stratified flow simulations remains an unsettled issue [12]. Furthermore, it is well-known in the literature that the LES-based flow characteristics are strongly dependent on the employed subgridscale (SGS) model, spatial resolution, filter type, etc. To circumvent these ad-hoc numerical influences, a viable alternative, i.e., the direct numerical simulation (DNS) approach, can be utilized. In this approach, the Navier-Stokes (NS) equations are solved without any averaging, any filtering, or any other approximations for turbulence. The true benefit of using the

25 DNS approach, lies in its ability to capture the small-scale turbulence structure in strongly stratified conditions. In recent years, a number of DNS studies have been conducted to investigate the fundamental characteristics of strongly stratified turbulent channel flows. Research topics include, but not limited to, criteria for turbulence decaying [12, 13, 14], impact of stratification on turbulence structures $[15,16,17,18]$, spatio-temporal characteristics 
of intermittent turbulence [19, 20, 21, 22], similarity theories [18, 21, 23, 24].

Despite the benefits afforded by DNS for analyses of very stably stratified flows, the Reynolds number $(R e)$ in DNS studies is quite limited due to the exorbitant computational cost. To the best of the author's knowledge, so far, the highest Re reported in DNS of stably stratified channel flows is on the order of $10^{4}[12,17,18,21,23,24]$, significantly lower than the typical $R e$ in the ABL ( $10^{7}$ or more). A critical question is: Is the low-Re DNSbased results relevant to the atmospheric flows? According to the Re-similarity theory in Townsend [25], the low-Re DNS simulations can reproduce the atmospheric flow conditions as long as the Re is high enough. Recent DNS studies have found encouraging indications of the Re-similarity theory by comparing the DNS-based and observational-based similarity relationships [21, 23, 24]. However, due to the limitation of $R e$, the range of stabilities is relatively short in those DNS studies. For example, the highest stability parameter $(z / \Lambda$ with $z$ being the height and $\Lambda$ being the local Obukhov length) reported in van de Wiel et al. [23], and He and Basu [24] is approximately 5, much lower than the typical values observed in the very stable ABL $(z / \Lambda$ on the order of 10 s, e.g., see Sorbjan and Grachev [26]). In 45 order to probe the very stably stratified turbulent flows using the DNS-based results, there is a need to push $R e$ to a higher level $\left(10^{5}\right.$ or more). Fortunately, with the tremendous advance in computational techniques, such large-scale DNS simulations can be performed using the petascale computer system (systems capable of performing $10^{15}$ or more operations per second). In terms of accuracy, the spectral method is considered to be the most efficient 50 option for DNS [27, 28]. However, the ability to handle complicated geometric boundary [29], e.g., complex topography, makes the finite-difference DNS approach a competitive option. It it noted that finite-difference DNS of stably stratified channel flows at petascale raises a number of issues.

- Fractional time-step method [30, 31] has found widespread usage in finite-difference DNS of channel flows. In order to eliminate the numerical viscous stability restriction, the semi-implicit scheme, i.e., explicit treatment for convective terms and implicit treatment for viscous terms, is a favorable choice for time advancement. The viscous stability restriction can be quite severe, especially in high $R e$ simulations where dense near-wall grids are used, and in strongly stratified flows where drastically nonstationary and bursting conditions are present, e.g., wave-motions, intermittent turbulence. For the second order spatial schemes, the resulting discretization equations can be 
efficiently solved using the factorization approximation [31]; however, the extension of factorization for higher order of spatial accuracy is cumbersome (see detailed discussion in Section 2). In recent years, a few high-order finite-difference DNS solvers have been developed for simulating turbulent channel flows [21, 32, 33]; however, all of them used an explicit time-advancement scheme. In order to enhance the numerical stability (decrease the computational costs), there is a need to develop a high order finite-difference DNS solver using semi-implicit scheme for simulating strongly stratified flows at high Re.

70

75 DNS solver for simulating strongly stratified turbulent channel flows at relatively high $R e$. To be more specific, in this work, a semi-implicit scheme is utilized for time-advancement, and the spatial derivatives are discretized using the fourth-order central differential scheme in the horizontal direction and the second-order central differential scheme in the vertical temperature, and pressure equations, are then directly solved using the Fourier analysis approach. The 2D domain decomposition technique is utilized for parallel communication, and the scalability of the newly developed DNS solver is tested with up to $32768 \mathrm{CPU}$ cores. The DNS solver is verified using the benchmark data in Moser et al. [37], and García-Villalba 90 and del Álamo [17] for neutrally and stably stratified channel flows. Moreover, two stably stratified open-channel simulations are conducted and analyzed with the highest Reynolds number being approximately $10^{5}$. We would like to highlight that the current DNS solver 
uses a non-slip boundary condition at the bottom wall to mimic the sheared boundary layer flows, which is different from the massively parallel DNS solvers for isotropic stratified turbulent flows, such as those reported in Rosenberg et al. [38], and de Bruyn Kops [39], to name a few.

The structure of this paper is as follows: in Section 2, we introduce the details of numerical method and summarize the computational configurations. The simulated profiles of mean flows and turbulence statistics are shown in Section 3. In this section, we also discuss the spatio-temporal characteristics of strongly stratified flows. Moreover, the simulated similarity relationships are compared with the observational-based ones to evaluate the relevance of the DNS-based results. Conclusion and future directions are given in Section 4. Finally, the verification of the newly developed DNS solver is presented in the Appendix.

\section{Method}

\subsection{Governing equations}

Under the Boussinesq approximation, the governing Navier-Stokes and the temperature equations for incompressible flows are presented in Equations 1-3. The governing equations are normalized by $h$ and $u_{\tau}$, where $h$ is the channel height, and $u_{\tau}=\sqrt{\tau_{w} / \rho_{0}}$ is the friction velocity with $\rho_{0}$ being the reference density and $\tau_{w}$ being the shear stress at walls. The friction Reynolds number and Richardson number are defined as $R e_{\tau}=u_{\tau} h / \nu$ and $R i_{\tau}=$ $\left(\theta_{\text {top }}-\theta_{\text {bot }}\right) g h / \theta_{\text {top }} u_{\tau}^{2}$, respectively. Here $\nu$ is the kinematic viscosity, $g$ is the gravitational acceleration, and the subscripts top and bot represent the top and bottom walls, respectively. We consider stably stratified air flows, so the Prandtl number $(P r)$ is set to 0.7. $\Delta P$ is the streamwise pressure gradient driving the flow and $p$ is the dynamic pressure.

$$
\begin{gathered}
\frac{\partial u_{j}}{\partial x_{j}}=0 \\
\frac{\partial u_{i}}{\partial t}+\frac{\partial\left(u_{i} u_{j}\right)}{\partial x_{j}}=\frac{1}{R e_{\tau}} \frac{\partial}{\partial x_{j}}\left(\frac{\partial u_{i}}{\partial x_{j}}\right)-\frac{\partial p}{\partial x_{i}}+\Delta P \delta_{i 1}+R i_{\tau} \theta \delta_{i 3} \\
\frac{\partial \theta}{\partial t}+\frac{\partial\left(\theta u_{j}\right)}{\partial x_{j}}=\frac{1}{R e_{\tau} \operatorname{Pr}} \frac{\partial}{\partial x_{j}}\left(\frac{\partial \theta}{\partial x_{j}}\right)
\end{gathered}
$$


Following Kim and Moin [31], the fractional time step method is utilized for the time advancement. First, the momentum equation (Eq. 2) is discretized by a semi-implicit scheme in time. The Crank-Nicolson scheme is used for the viscous terms. For the convective terms, either Adams-Bashforth or Runge-Kutta scheme can be used. For easy presentation, the second order Adams-Bashforth scheme is presented here and the detailed implementation of the Runge-Kutta scheme can be seen in Orlandi [40]. An intermediate velocity field, e.g., $u_{i}^{*}$, is then calculated based on the flow fields from the current $(n)$ and previous $(n-1)$ time steps:

$$
u_{i}^{*}=u_{i}^{n}+\frac{1}{2} \Delta t\left(3 N_{i}^{n}-N_{i}^{n-1}\right)+\frac{\Delta t}{2 R e_{\tau}} L\left(u_{i}^{*}+u_{i}^{n}\right)-\Delta t \frac{\delta p^{n}}{\delta x_{i}} .
$$

Here $N_{i}$ is the discretization of the nonlinear convective terms, i.e., $-\frac{\delta}{\delta x_{j}}\left(u_{i} u_{j}\right)$. The external 125 body force terms, i.e., $\Delta P \delta_{i 1}$ and $R i_{\tau} \theta \delta_{i 3}$, are also included in $N_{i}$. The discretization of the linear viscous terms is given by $L\left(u_{i}\right)=\frac{\delta}{\delta x_{j}}\left(\frac{\delta u_{i}}{\delta x_{j}}\right)$. Note that the resulting intermediate velocity $u_{i}^{*}$ does not satisfy the continuity equation (Eq. 1). To enforce continuity, a pseudo pressure $(\phi)$ is defined to "project" $u_{i}^{*}$ to a divergence-free velocity field $\left(u_{i}^{n+1}\right)$ :

$$
\frac{u_{i}^{n+1}-u_{i}^{*}}{\Delta t}=-\frac{\delta \phi}{\delta x_{i}} .
$$

Taking the divergence of Eq. 5 and enforcing $\frac{\delta u_{i}^{n+1}}{\delta x_{i}}=0$, one obtains the pressure Poisson equation:

$$
\frac{\delta}{\delta x_{i}}\left(\frac{\delta \phi}{\delta x_{i}}\right)=\frac{1}{\Delta t} \frac{\delta u_{i}^{*}}{\delta x_{i}}
$$

Solving Eq. 6 for $\phi$, and substituting it into Eq. 5, one obtains a new velocity field $u_{i}^{n+1}$ at time step $n+1$ which satisfies both continuity and momentum equations. The pressure at the new time step is updated by:

$$
p^{n+1}=p^{n}+\phi-\frac{\Delta t}{2 R e_{\tau}} L(\phi) .
$$

For the temperature equation (Eq. 3), a similar semi-implicit scheme is utilized for time advancement:

$$
\theta^{n+1}=\theta^{n}+\frac{1}{2} \Delta t\left(3 N^{n}-N^{n-1}\right)+\frac{\Delta t}{2 \operatorname{Re} e_{\tau} \operatorname{Pr}} L\left(\theta^{n+1}+\theta^{n}\right),
$$




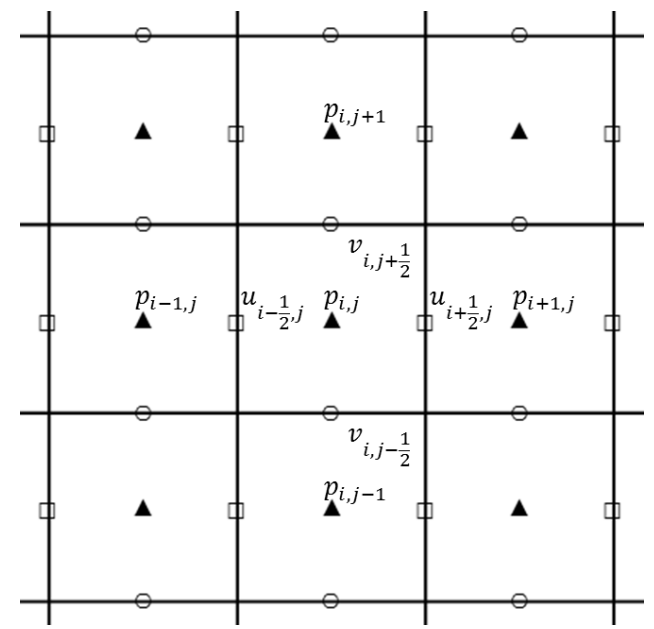

Figure 1: Schematic of fully staggered grids. A $2 \mathrm{D}$ case is shown for reference. Here $u$ and $v$ are stored at the cell surfaces while $p$ is stored in the cell centers.

where $N=-\frac{\delta}{\delta x_{j}}\left(\theta u_{j}\right)$ is the discretization of the nonlinear convective terms.

\subsection{Spatial discretization}

Spatial derivatives in Eqs. 4 to 8 are discretized on fully staggered grids [41] for a strong coupling of velocity and pressure fields, as illustrated in Fig. 1. Note that a 2D staggered grid schematic is shown for reference. Here $u$ and $v$ represent the velocity components in the $x$ and $y$ directions, respectively. The velocity components $(u, v)$ are stored at the cell surfaces, whereas the pressure and temperature are stored in the cell centers. For channel flow simulations, the horizontal grids are uniformly spaced, while in the wall-normal direction, a stretched grid is used for a better representation of flows near the walls. The horizontal and vertical derivatives are discretized using the fourth order central differential scheme and the second order central differential scheme, respectively.

Specifically, in the horizontal direction, the nonlinear convective terms are casted in the divergence form and then discretized using the fourth order central differential scheme. For example, for the $\frac{\delta(u u)}{\delta x}$ and $\frac{\delta(u v)}{\delta y}$ terms:

$$
\frac{\delta(u u)_{i+\frac{1}{2}, j, k}}{\delta x}=\frac{-u_{i+2, j, k}^{2}+27 u_{i+1, j, k}^{2}}{24 \Delta x}+\frac{-27 u_{i, j, k}^{2}+u_{i-1, j, k}^{2}}{24 \Delta x} .
$$




$$
\frac{\delta(u v)_{i+\frac{1}{2}, j, k}}{\delta y}=\frac{-(u v)_{i+\frac{1}{2}, j+\frac{3}{2}, k}+27(u v)_{i+\frac{1}{2}, j+\frac{1}{2}, k}}{24 \Delta y}+\frac{-27(u v)_{i+\frac{1}{2}, j-\frac{1}{2}, k}+(u v)_{i+\frac{1}{2}, j-\frac{3}{2}, k}}{24 \Delta y}
$$
error produced by the convective discretization scheme was found to be significantly (three to four orders of magnitude) smaller than that dissipated by viscosity in DNS [33].

For the viscous terms and the pressure term, the fourth order central differential scheme is straightforward to implement and no extra interpolation is needed. For example, for the $170 \frac{\partial}{\partial x}\left(\frac{\partial u}{\partial x}\right)$ term in $u$ equation:

$$
\frac{\delta}{\delta x}\left(\frac{\delta u_{i, j, k}}{\delta x}\right)=\frac{-u_{i+2, j, k}+16 u_{i+1, j, k}-30 u_{i, j, k}+16 u_{i-1, j, k}-u_{i-2, j, k}}{12(\Delta x)^{2}} .
$$


In the vertical direction, a stretched grid is used, and the variation of grid points is defined by the following hyperbolic tangent function:

$$
z_{i}=h-\frac{h}{\tanh (2 \alpha)} \tanh \left(\frac{2 \alpha}{1-N_{1}}\left(i-N_{1}\right)\right),
$$

where $\alpha$ is a parameter to control the refinement of grids near the wall and $N_{1}$ is the grid number in the vertical direction. For the vertical derivatives, the second order central differential scheme is used, for example:

$$
\begin{gathered}
\frac{\delta u_{i+\frac{1}{2}, j, k}}{\delta z}=\frac{u_{i+\frac{1}{2}, j, k+\frac{1}{2}}-u_{i+\frac{1}{2}, j, k-\frac{1}{2}}}{\Delta z(k)}, \\
\frac{\delta^{2} u_{i+\frac{1}{2}, j, k}}{\delta z^{2}}=\frac{\frac{u_{i+\frac{1}{2}, j, k+1}-u_{i+\frac{1}{2}, j, k}}{\Delta z_{t}(k)}-\frac{u_{i+\frac{1}{2}, j, k}-u_{i+\frac{1}{2}, j, k-1}}{\Delta z_{b}(k)}}{\Delta z(k)},
\end{gathered}
$$

where $\Delta z_{t}(k)=\frac{\Delta z(k)+\Delta z(k+1)}{2}$ and $\Delta z_{b}(k)=\frac{\Delta z(k)+\Delta z(k-1)}{2}$. The velocity $u_{i+\frac{1}{2}, j, k+\frac{1}{2}}$ can be obtained by linear interpolation in the $z$ direction.

\subsection{Solution of the momentum, temperature, and pressure equations}

As can be see in Eq. 4, to obtain the intermediate velocity $u^{*}$, one needs to solve the Helmholz equation in the following form:

$$
u^{*}-\frac{\Delta t}{2 R e_{\tau}} L\left(u^{*}\right)=u^{n}+\frac{1}{2} \Delta t\left(3 N^{n}-N^{n-1}\right)+\frac{\Delta t}{2 R e_{\tau}} L\left(u^{n}\right)-\Delta t \frac{\delta p^{n}}{\delta x}=Q^{u} .
$$

Substituting the spatial discretization in Section 2.3 into Eq. 17, one obtains the linear algebraic equations for $u^{*}$. As mentioned in the introduction, the factorization approximation, introduced in the original fractional time step approach in Kim and Moin [31], was shown to be quite efficient in solving the linear algebraic equations for the second order spatial discretization. However, for the fourth order spatial schemes, the factorization approximation method becomes hard to implement since the factorization still results in a five diagonal matrix in the $x$ and $y$ directions, which is quite cumbersome to inverse. To mitigate this issue, in this paper, the Fourier analysis approach is utilized to solve the Helmholz equation for velocity fields. Let the intermediate velocity $\left(u^{*}\right)$ and the source term $\left(Q^{u}\right)$ be represented 190 by the discrete Fourier transform:

$$
u_{i, j, k}^{*}=\sum_{l=0}^{N_{x}-1} \sum_{m=0}^{N_{y}-1} \tilde{u}_{l, m, k}^{*} W_{x}^{i l} W_{y}^{j m},
$$




$$
Q_{i, j, k}^{u}=\sum_{l=0}^{N_{x}-1} \sum_{m=0}^{N_{y}-1} \tilde{Q}_{l, m, k}^{u} W_{x}^{i l} W_{y}^{j m},
$$

defined in $i=1,2, \ldots, N_{x}, j=1,2, \ldots, N_{y}$. Here $N_{x}$ and $N_{y}$ are the cell numbers in the $x$ and $y$ directions, respectively, and $W_{x}=e^{I 2 \pi / N_{x}}, W_{y}=e^{I 2 \pi / N_{y}}$ with $I=\sqrt{-1}$. Substituting Eqs. 18 and 19 into Eq. 17, one obtains a tridiagonal system of equations, which can be easily solved by Thomas algorithm:

$$
A \tilde{u}_{l, m, k-1}^{*}+B \tilde{u}_{l, m, k}^{*}+C \tilde{u}_{l, m, k+1}^{*}=\tilde{Q}_{l, m, k}^{u} .
$$

Here the coefficients are given by: $A=-\frac{\Delta t}{2 R e_{\tau} \Delta z(k) \Delta z_{b}(k)}, C=-\frac{\Delta t}{2 R e_{\tau} \Delta z(k) \Delta z_{t}(k)}$, $B=1-k_{x}-k_{y}-A-C$, and $k_{x}=\frac{\Delta t\left(-W_{x}^{2 l}+16 W_{x}^{l}-30+16 W_{x}^{-l}-W_{x}^{-2 l}\right)}{24 R e_{\tau}(\Delta x)^{2}}, \quad k_{y}=$ $\frac{\Delta t\left(-W_{y}^{2 m}+16 W_{y}^{m}-30+16 W_{y}^{-m}-W_{y}^{-2 m}\right)}{24 R e_{\tau}(\Delta y)^{2}}$ are the modified wavenumbers. $u^{*}$ can then be calculated using the inverse discrete Fourier transform of $\tilde{u}^{*}$. The Fourier analysis approach can also be applied for the $v, w$, and $T$ equations, which results in similar coefficients and modified wavenumbers.

Once the intermediate velocity fields are known, one can solve the pressure Poisson equation (Eq. 6) for the pseudo pressure $(\phi)$. Similar to that shown in Section 2.3, the Poisson equation is discretized using the fourth order and the second order central differential schemes in the horizontal and vertical directions, respectively. As a result, a fifteen-point stencil is obtained for the Poisson equation. By utilizing a similar Fourier analysis and substituting the corresponding discrete Fourier transform into Eq. 6, the following tridiagonal system of equations are obtained for $\phi$ :

$$
A \tilde{\phi}_{l, m, k-1}+B \tilde{\phi}_{l, m, k}+C \tilde{\phi}_{l, m, k+1}=\tilde{Q}_{l, m, k}^{p},
$$

where $A=\frac{1}{\Delta z(k) \Delta z_{b}(k)}, C=\frac{1}{\Delta z(k) \Delta z_{t}(k)}, B=k_{x}+k_{y}-A-C$, and $k_{x}=\left(W_{x}^{3 l}-54 W_{x}^{2 l}+\right.$ $\left.783 W_{x}^{l}-1460+783 W_{x}^{-l}-54 W_{x}^{-2 l}+W_{x}^{-3 l}\right) / 576(\Delta x)^{2}, k_{y}=\left(W_{y}^{3 m}-54 W_{y}^{2 m}+783 W_{y}^{m}-\right.$ $\left.1460+783 W_{y}^{-m}-54 W_{y}^{-2 m}+W_{y}^{-3 m}\right) / 576(\Delta y)^{2}$ are the modified wavenumbers. Once the pseudo pressure is obtained, the pressure and velocity fields at the new time step can be calculated using Eqs. 7 and 5, respectively.

As shown above, a generalized Fourier analysis approach is used for solving the velocity and temperature Helmholz equations, as well as the pressure Poisson equation. We would like to highlight that an important advantage of using the generalized Fourier solver is 
that the implementation for massive parallelism is quite straightforward (please see detailed discussion in Section 2.6).

\subsection{Boundary conditions}

In the DNS of open-channel flows, the computational domain is a rectangular box. The domain is periodic in the horizontal direction and is bounded by two walls in the vertical direction. Periodic boundary conditions are prescribed along the streamwise $(x)$ and spanwise $(y)$ boundaries. A non-slip boundary condition $(u, v, w=0)$ is specified at the bottom wall $(z=0)$, whereas at the top wall $(z=h)$, a free-slip boundary condition is imposed $(\partial u / \partial z=0, \partial v / \partial z=0, w=0)$. In order to impose stratification in the channel, fixed normalized temperatures $\left(\theta_{\mathrm{top}}=0, \theta_{\mathrm{bot}}=-1\right)$ are also prescribed along the top and the bottom walls. The pressure boundary condition at the bottom and top walls is zero-gradient $(\partial p / \partial z=0)$.

Similar boundary conditions are also needed when solving the velocity, temperature, and pressure equations using the Fourier analysis method. For example, for Eq. 20, the same non-slip and free-slip boundary conditions are applied for the Fourier coefficients $\tilde{u}^{*}$, i.e., $\tilde{u}^{*}=0$ at the bottom wall and $\partial \tilde{u}^{*} / \partial z=0$ at the top wall. For the temperature equation, the fixed temperature condition is imposed by keeping the Fourier coefficient $\tilde{\theta}_{l=0, m=0}=-1$ and other Fourier coefficients to be zero at the bottom wall (similar treatment is applied for the top wall). For the pressure Poisson equation, we set $\tilde{p}_{l=0, m=0}=0$ at the bottom wall to remove the null space. No such treatment is needed for the velocity and temperature Helmholz equations.

As shown in Eq. 2, a streamwise pressure difference $(\Delta P)$ is imposed in the $u$ equation to drive the flow. A constant friction condition can be imposed if the pressure difference is set to be invariant during the simulations, i.e., $\Delta P=1$. Alternatively, $\Delta P$ can vary during the simulation to adjust a constant mass flow rate. As discussed in Brethouwer et al. [20], compared with the constant pressure difference approach, the constant mass flow rate condition has a better performance in reducing low-frequency temporal oscillations for the very stable cases, especially if a small computational domain is used. However, its limitation is that the friction is not controlled and the grid resolution in wall-unit is not known in advance. This can be an issue for the finite-difference solver since a relatively fine grid resolution is needed. Given this motivation, the constant pressure difference condition is used in this study. 


\section{6. $2 D$ domain decomposition for parallel computation}

In this study, a 2D "pencil" domain decomposition method is used for parallel compu-

(a)

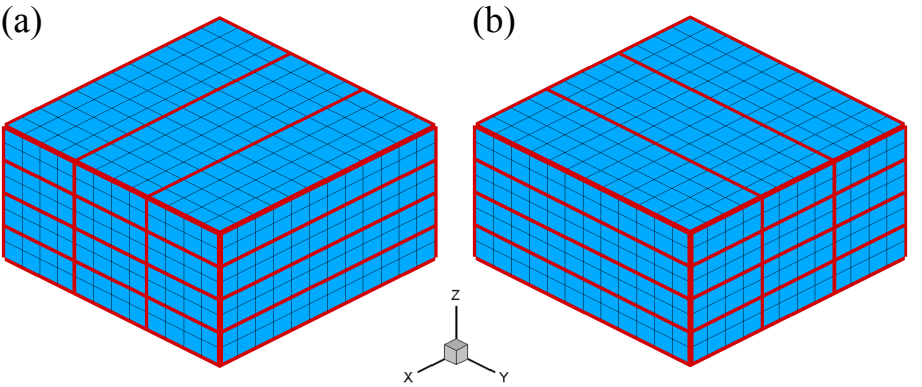

(c)

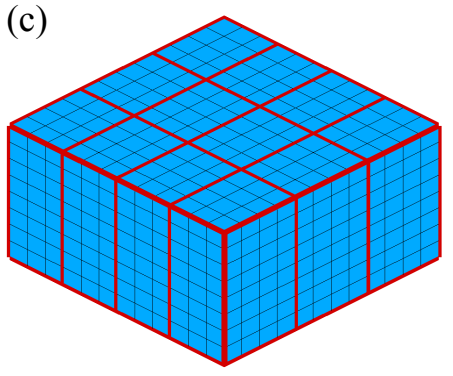

Figure 2: Illustration of 2D pencil decomposition and transposition for the computational domain. (a), (b), and (c) are for the $\mathrm{X}, \mathrm{Y}$, and $\mathrm{Z}$ pencil arrangements, respectively. Here the computational domain is divided into $12 \times 12 \times 8$ cells (black thin lines) in the $x, y$, and $z$ directions, respectively. The domain is then decomposed into 4 rows and 3 columns $\left(P_{\text {row }}=4, P_{\text {col }}=3\right.$; red thick lines) in the $\mathrm{Z}$ pencil arrangement for parallel simulations. The $\mathrm{Z}$ pencil arrangement can be further transposed to the $\mathrm{Y}$ or $\mathrm{X}$ pencil for parallel communication.

We present the detailed implementation of the 2D decomposition in our DNS solver by showing a simplified example in Fig. 2. Here the computational domain is divided into $12 \times 12 \times 8$ cells (black thin lines) in the $x, y$, and $z$ directions, respectively. The domain is then decomposed into 4 rows and 3 columns of pencils ( $P_{\text {row }}=4, P_{\text {col }}=3$; red thick lines), so that each Message Passing Interface (MPI) process can work on one pencil in parallel. Fig. 3 summaries the flow chart of the current DNS solver using the 2D pencil decomposition technique. Initially, the domain is divided using the $Z$ pencil arrangement (Fig. 2c). Parallel communication is needed when: (i) calculating the spatial discretization for the convective, viscous, pressure terms, etc; (ii) performing the 2D Fast Fourier Transform (FFT), e.g., Eqs. 18 and 19, for the solution of the velocity, temperature, and pressure equations. For 
the calculation of spatial discretization, standard MPI blocking communication is used in the horizontal direction between each pencil. In order to perform 2D FFT at horizontal planes, the $Z$ pencil arrangement is transposed to $Y$ pencil arrangement (Fig. 2b) and 1D FFT is

$$
\text { is quite straightforward and one does not need to change the original serial coding logic. }
$$

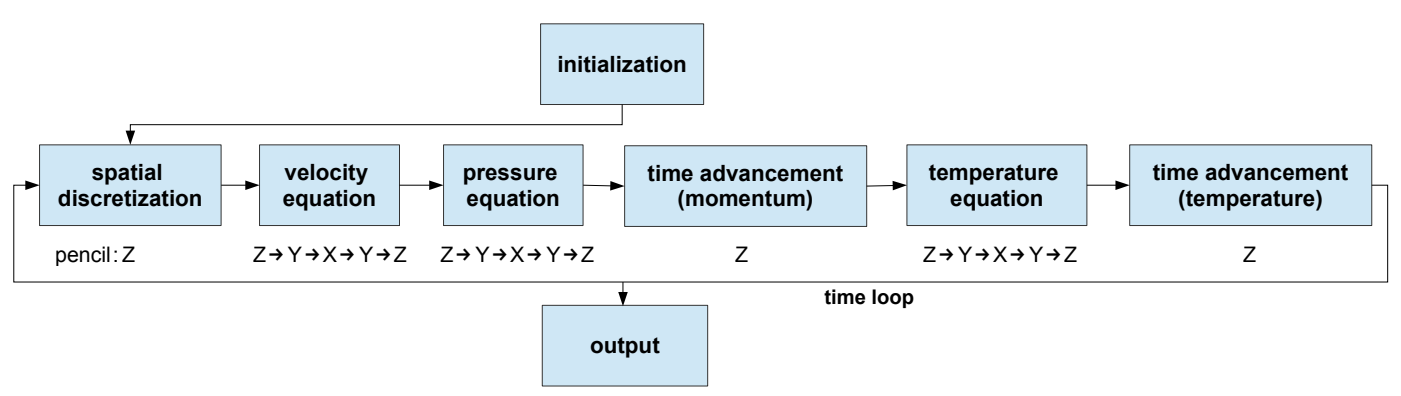

Figure 3: Flow chart of the current DNS solver using the 2D pencil decomposition technique. The pencil transposition operations at each step are also shown for reference.

As shown above, the data transposition is a critical step for parallel communication. Essentially, the data transposition is implemented through the MPI_ALLTOALL function. In this function, all the data assigned to the MPI processes are globally exchanged to each other. It is clear that this operation can cause significant communication delay, and one should minimize its usage for a better parallel efficiency. As shown in Fig. 3, a total of 12 transpositions are needed for each time-iteration. It is also noted that the efficient $2 \mathrm{D}$ data transposition using the MPI_ALLTOALL function is probably a purely computer-science related topic, and its detailed implementation can be quite cumbersome. Fortunately, at present, several high-performance data transposition libraries are readily available, e.g., P3DFFT [45], 2DECOMP\&FFT [46]. In the current DNS solver, the 2D transposition is performed using the 2DECOMP\&FFT library v1.5.847 and the FFT is conducted with the FFTW library v3.3.4 [47]. 
Table 1: Evaluation of the strong scalability of the current DNS solver on ARL HPC Excalibur, AFRL HPC Lightning, and TACC Stampede. The parallel efficiency is calculated as $E_{1}=\frac{P_{0}}{P} \frac{T_{0}}{T}$. Here $P$ and $T$ are the number of cores and the elapsed time per step, respectively. The subscript 0 denotes the case using smallest number of cores.

\begin{tabular}{|c|c|c|c|c|c|c|c|c|c|c|c|c|}
\hline \multicolumn{13}{|c|}{ Excalibur } \\
\hline Grids & \multicolumn{4}{|c|}{$2048 \times 2048 \times 256$} & & \multicolumn{4}{|c|}{$4096 \times 4096 \times 512$} & \multicolumn{3}{|c|}{$8192 \times 8192 \times 512$} \\
\hline Cores & 512 & 1024 & 2048 & 4096 & & 4096 & 8192 & 1638 & & 16384 & 32768 & \\
\hline Time/Step (s) & 13.58 & 7.00 & 3.55 & 1.70 & & 17.18 & 8.58 & 4.13 & & 28.17 & 14.86 & \\
\hline \multirow[t]{2}{*}{ Efficiency } & $100 \%$ & $97 \%$ & $96 \%$ & $100 \%$ & & $100 \%$ & $100 \%$ & $104 \%$ & & $100 \%$ & $95 \%$ & \\
\hline & \multicolumn{9}{|c|}{ Lightning } & & & \\
\hline Grids & \multicolumn{5}{|c|}{$1024 \times 1024 \times 128$} & \multicolumn{4}{|c|}{$2048 \times 2048 \times 256$} & \multicolumn{3}{|c|}{$4096 \times 4096 \times 512$} \\
\hline Cores & 128 & 256 & 512 & 1024 & 2048 & 512 & 1024 & 2048 & 4096 & 4096 & 8192 & 16384 \\
\hline Time/Step (s) & 4.12 & 2.29 & 1.15 & 0.55 & 0.32 & 9.99 & 5.12 & 2.72 & 1.57 & 14.17 & 6.72 & 4.34 \\
\hline Efficiency & $100 \%$ & $90 \%$ & $90 \%$ & $94 \%$ & $80 \%$ & $100 \%$ & $98 \%$ & $92 \%$ & $80 \%$ & $100 \%$ & $105 \%$ & $82 \%$ \\
\hline \multicolumn{13}{|c|}{ Stampede } \\
\hline Grids & \multicolumn{5}{|c|}{$1024 \times 1024 \times 128$} & \multicolumn{4}{|c|}{$2048 \times 2048 \times 256$} & \multicolumn{3}{|c|}{$4096 \times 4096 \times 512$} \\
\hline Cores & 128 & 256 & 512 & 1024 & 2048 & 512 & 1024 & 2048 & 4096 & 4096 & 8192 & 16384 \\
\hline Time/Step (s) & 3.76 & 1.89 & 1.06 & 0.52 & 0.30 & 8.07 & 4.77 & 2.41 & 1.30 & 10.80 & 6.18 & 3.40 \\
\hline Efficiency & $100 \%$ & $99 \%$ & $89 \%$ & $90 \%$ & $78 \%$ & $100 \%$ & $85 \%$ & $84 \%$ & $78 \%$ & $100 \%$ & $87 \%$ & $79 \%$ \\
\hline
\end{tabular}

\subsection{Parallel efficiency}

The parallel efficiency, or the scalability, is essential for large-scale DNS simulations. The current DNS solver has been tested on the following HPC platforms: ARL HPC Excalibur, AFRL HPC Lightning, and TACC Stampede. Excalibur is a Cray XC40 system equipped with Intel Xeon E5-2698 v3 processors clocked at $2.3 \mathrm{GHz}$. The total number of nodes

295 in Excalibur is 3098 with $32 \mathrm{CPU}$ cores per node, and they are interconnected using the Cray Aries with Dragonfly topology. Excalibur has a peak PFLOPS of 3.7. Lightning is a Cray XC30 system populated with Xeon E5-2697 v2 processors clocked at $2.7 \mathrm{GHz}$. In total, Lightning has 2370 computational nodes with 24 cores per node, connected through the Cray Aries network. Lightning is rated at 1.2 peak PFLOPS. Stampede is a Dell Linux soo Cluster configured with more than 6400 nodes, and each is outfitted with two Intel Xeon E5-2680 processors clocked at $2.7 \mathrm{GHz}$. The Mellanox FDR InfiniBand technology is used for interconnection. The peak PFLOPS of Stampede is approximately 2.

First, the strong scalability of the current DNS solver is tested. The strong scalability is defined as the variation of the simulation speed (elapsed time per step) when increasing ${ }_{305}$ the CPU cores for a fixed grid number. A perfect strong scalability implies that simulation 
speed increases by a factor of two if the CPU cores are doubled. Table 1 shows the strong scalability test results using the three HPC systems. Here four stably stratified open-channel flow cases, with the grid points being $0.13,1.1,8.6$, and 34.4 billion, are simulated using various numbers of CPU cores. The parallel efficiency for strong scalability is calculated as $E_{1}=\frac{P_{0}}{P} \frac{T_{0}}{T}$. Here $P$ and $T$ are the number of cores and the elapsed time per step, respectively. The subscript 0 denotes the case using smallest number of cores. Overall, the current DNS solver manifests reasonably good parallel efficiency on these three HPC platforms with Excalibur having the best performance. Selectively, the scalability data for Excalibur is shown in Fig. 4 along with the ideal scalability line for reference. Remarkably, we observe a 95\% parallel efficiency when increasing the CPU cores from 16384 to 32768 for the 34.4 billion grid case. This high parallel efficiency is probably attributed to the dragonfly topology employed in the Cray Aries interconnection, which provides significantly improved bandwidth for the MPI_ALLTOALL function. However, it is also noted that for a given number of grids and CPU cores, the simulation speed for Excalibur is the slowest among the three HPC platforms. This is expected since Excalibur has $32 \mathrm{CPU}$ cores per node, as a trade-off, the CPU frequency is relatively low $(2.3 \mathrm{GHz})$. We would like to point out that a few super-linearity scalability results are observed in Table 1 (parallel efficiency is slightly larger than 100\%). Similar super-linearity results were also previously reported in the scalability tests in Laizet and $\mathrm{Li}$ [32], and Lee et al. [48]. This is not unexpected given the fact that the parallel efficiency is known to be sensitive to the performance of network and the 2D mapping strategy, i.e., the choice of $P_{\text {row }}$ and $P_{\text {col }}$. The optimal values for $P_{\text {row }}$ and $P_{\text {col }}$ vary depending on the architecture of the HPC platforms, as well as the interconnection layout. In principle, $P_{\text {row }}$ should not be much larger than $P_{\text {col }}$ to ensure an efficient usage of memory cache for distributed 3D arrays (please refer to Laizet and Li [32] for detailed discussion). However, in practice, some trial and errors are needed to ensure a maximal parallel efficiency.

In addition to the strong scalability, the weak scalability is tested (Table 2). The weak scalability is defined as the variation of the simulation speed when increasing the CPU cores with the number of grids per core fixed. In this paper, the number of grids per core is set to be approximately 1 million. In other words, the grid number for the 128-core case is approximately 0.54 billion, whereas for the 16384-core case, the grid number is about 17.2 billion. One can see that, with increasing the number of cores, the elapsed time per 
Table 2: Evaluation of the weak scalability of the current DNS solver on ARL HPC Excalibur, AFRL HPC Lightning, and TACC Stampede. The number of grid per core is set to be approximately 1 million. The parallel efficiency is calculated as $E_{2}=\frac{T_{0}}{T}$. Here $T$ is the the elapsed time per step. The subscript 0 denotes the case using smallest number of cores.

\begin{tabular}{lllllll}
\hline & \multicolumn{7}{c}{ Excalibur } \\
\hline Cores & 512 & 1024 & 2048 & 4096 & 8192 & 16384 \\
\hline Time/Step (s) & 6.91 & 7.00 & 7.48 & 7.93 & 7.88 & 7.30 \\
\hline Efficiency & $100 \%$ & $99 \%$ & $92 \%$ & $87 \%$ & $88 \%$ & $95 \%$ \\
\hline \multicolumn{7}{c}{ Lightning } \\
\hline Cores & 512 & 1024 & 2048 & 4096 & 8192 & 16384 \\
\hline Time/Step (s) & 5.89 & 5.40 & 5.93 & 6.68 & 6.44 & 6.99 \\
\hline Efficiency & $100 \%$ & $109 \%$ & $99 \%$ & $88 \%$ & $91 \%$ & $84 \%$ \\
\hline & & \multicolumn{5}{c}{ Stampede } \\
\hline Cores & 512 & 1024 & 2048 & 4096 & 8192 & 16384 \\
\hline Time/Step (s) & 4.08 & 4.24 & 4.42 & 5.14 & 5.22 & 5.84 \\
\hline Efficiency & $100 \%$ & $96 \%$ & $92 \%$ & $79 \%$ & $78 \%$ & $70 \%$ \\
\hline
\end{tabular}

step globally decreases. This is expected since the increasing grid number causes excessive network communication which consequently decreases the parallel efficiency. Overall, the weak scalability performance is quite reasonable with Excalibur having the best parallel efficiency.

Given the good scalability, the current DNS solver is shown to be competent to perform petascale DNS simulations for stratified channel flows with grid number on the order of 10 billion. In order to further test its ability to capture the turbulence characteristics of strongly stratified flows, two DNS simulations are conducted and analyzed in detail at a relatively high $R e$ in the following sections.

\subsection{Simulation configurations}

Table 3 summaries the simulation configurations used in the study. Two stably stratified open-channel simulations (S1: weakly stratified, $R i_{\tau}=100 ; \mathrm{S} 2$ : strongly stratified, $R i_{\tau}=$ $\left.10^{4}\right)$ are performed at $R e_{\tau}=1000$ for detailed analyses. For the strongly stratified case, the bulk Reynolds number, i.e., $\operatorname{Re}_{b}=u_{b} h / \nu$, is about $10^{5}$, and the corresponding bulk Richardson number, i.e., $R i_{b}=\left(\theta_{\text {top }}-\theta_{b o t}\right) g h / \theta_{t o p} u_{b}^{2}$, is approximately 1.0. Here the bulk velocity $u_{b}$ is defined as the averaged velocity in the channel. It is important to note that the 


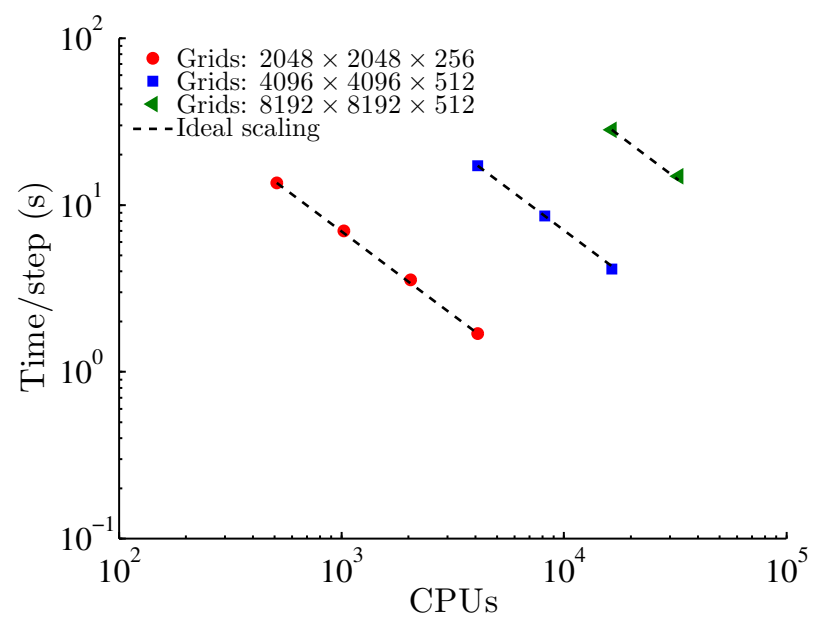

Figure 4: Scalability test for the current DNS solver on Excalibur. Platform: Cray XC40. CPU: Intel Xeon E5-2698 v3; Interconnection: Cray Aries / Dragonfly. Compiler: Intel Fortran v15.0.1; MPI library: Cray-MPICH v7.1.0.

open-channel flow is equivalent to a $z$-symmetrized (closed-channel) plane Poiseuille flow; however, the definitions of $R i_{\tau}$ in these two cases are slightly different. The conventional definition for $R i_{\tau}$ in closed-channel flows $[17,49]$ is two times larger than the definition used here for open-channel flows. In other words, the stratification level with $R i_{\tau}=10^{4}$ in an open-channel is similar to that in a closed-channel with $R i_{\tau}=2 \times 10^{4}$.

As mentioned above, the grids are uniformly spaced in the horizontal direction and stretched in the vertical. The grid resolutions are set to be $\Delta x^{+}=8.9$ and $\Delta y^{+}=4.9$, where $\Delta x^{+}$and $\Delta y^{+}$are the normalized grid resolutions in inner units (e.g., $\Delta x^{+}=\Delta x u_{\tau} / \nu$ ). To fully resolve turbulence in near-wall regions, the first grid level near the bottom wall is located at $z^{+}<0.4$, and more than 20 grids are set within $z^{+}<10$, where $z^{+}$is the normalized grid distance from the bottom wall, $z^{+}=z u_{\tau} / \nu$. The horizontal and vertical spatial-derivatives are discretized using the fourth order and second order central differential schemes, respectively, as shown in Section 2.3. For S1, the coupled Adams-Bashforth and Crank-Nicolson scheme is used for time advancement, whereas for S2 the coupled RungeKutta and Crank-Nicolson scheme is used in order to obtain better numerical stability. The boundary conditions for the stably stratified open-channel flows were introduced in 370 Section 2.5.

According to García-Villalba and del Álamo [17], Brethouwer et al. [20], and Ansorge 
Table 3: Summary of the computational configurations for the neutrally and stably stratified channel flows in this study. The cases S1 and S2 (open-channel configuration) are for detailed analyses, and the cases V1 to V7 (closed-channel configuration) are for solver verification. $R e_{b}$ and $R e_{\tau}$ are the bulk and the friction Reynolds numbers, respectively; $R i_{b}$ and $R i_{\tau}$ are the bulk and the friction Richardson numbers, respectively; $h / L$ is stability parameter with $L$ being the Obukhov length and $h$ being the open-channel height ( $h$ is set to be 1.0 for the current study); $L_{x}$ and $L_{y}$ are the domain lengths in the streamwise $(x)$ and spanwise $(y)$ directions, respectively; $N_{x}, N_{y}, N_{z}$ are the cell numbers in the $x, y, z$ directions, respectively; $\Delta x^{+}, \Delta y^{+}, \Delta z^{+}$are the normalized grid resolutions (e.g., $\Delta x^{+}=\Delta x u_{\tau} / \nu$ ). The smaller and larger $\Delta z^{+}$represent the vertical grid resolution at the bottom and the top walls, respectively. $t u_{\tau} / h$ is the non-dimensional averaging time for the calculations of turbulence statistics.

\begin{tabular}{lllllllllllllll}
\hline Case & $R e_{b}$ & $R e_{\tau}$ & $R i_{b}$ & $R i_{\tau}$ & $h / L$ & $L_{x}$ & $L_{y}$ & $N_{x}$ & $N_{y}$ & $N_{z}$ & $\Delta x^{+}$ & $\Delta y^{+}$ & $\Delta z^{+}$ & $t u_{\tau} / h$ \\
\hline S1 & 23200 & 1000 & 0.19 & 100 & 0.41 & $25 h$ & $10 h$ & 2816 & 2048 & 256 & 8.9 & 4.9 & $0.4-8.8$ & 10 \\
S2 & 100500 & 1000 & 0.99 & $10^{4}$ & 6.90 & $25 h$ & $10 h$ & 2816 & 2048 & 256 & 8.9 & 4.9 & $0.4-8.8$ & 5 \\
V1 & 2800 & 180 & 0 & 0 & 0 & $4 \pi h$ & $4 \pi h / 3$ & 256 & 192 & 128 & 8.8 & 4.4 & $0.3-6.1$ & 20 \\
V2 & 6900 & 395 & 0 & 0 & 0 & $2 \pi h$ & $\pi h$ & 288 & 288 & 288 & 8.8 & 4.3 & $0.3-6.1$ & 20 \\
V3 & 11000 & 590 & 0 & 0 & 0 & $2 \pi h$ & $\pi h$ & 432 & 432 & 432 & 8.6 & 4.3 & $0.3-6.1$ & 20 \\
V4 & 2800 & 180 & 0 & 0 & 0 & $8 \pi h$ & $4 \pi h$ & 512 & 512 & 128 & 8.8 & 4.4 & $0.3-6.3$ & 20 \\
V5 & 3100 & 180 & 0.03 & 18 & 0.06 & $8 \pi h$ & $4 \pi h$ & 512 & 512 & 128 & 8.8 & 4.4 & $0.3-6.3$ & 20 \\
V6 & 3800 & 180 & 0.13 & 120 & 0.23 & $8 \pi h$ & $4 \pi h$ & 512 & 512 & 128 & 8.8 & 4.4 & $0.3-6.3$ & 20 \\
V7 & 5400 & 180 & 0.27 & 480 & 0.64 & $8 \pi h$ & $4 \pi h$ & 512 & 512 & 128 & 8.8 & 4.4 & $0.3-6.3$ & 20 \\
\hline
\end{tabular}

and Mellado [21], a large computational domain is needed to accommodate the spatialtemporal variation of stably stratified flows, especially for very stable cases. In the current study, the domain size is chosen to be $L_{x} \times L_{y} \times L_{z}=25 h \times 10 h \times h$. In order to obtain initial conditions for the simulations, coarse-resolution cases are run for about 50 non-dimensional time (i.e., $t u_{\tau} / h \approx 50$ ) to obtain quasi-stationary flow fields. Then the flow variables are interpolated to fine-resolution initial fields. Finally, 10 and 5 non-dimensional times are run to calculate the turbulence statistics for S1 and S2, respectively. The S2 case took about 90 hours using 8192 cores on AFRL HPC Lightning.

In order to verify the current DNS solver, seven simulations (V1-V7, see Table 3) are performed for both neutrally and stably stratified flows and the simulations results are compared with the benchmark data reported in Moser et al. [37], and García-Villalba and del Álamo [17], as can be seen in the Appendix. The simulations results are shown to be in reasonably good agreement with the benchmark DNS data. These results demonstrate 385 the success of the current DNS solver and build confidence for the simulations and analyses 
reported in the rest of the paper.

\section{Results and Discussion}

The simulation results for S1 and S2 are reported in this section to demonstrate the ability of the newly developed DNS solver for simulating stably stratified channel flows at a buoyancy, resulting in significant modification of turbulence structures compared with the neutral case. Such topic has been investigated in a number of LES and DNS studies, e.g., Armenio and Sarkar [49], and García-Villalba and del Álamo [17], based on stratified closed-channel flows at relatively low Reynolds numbers $\left(R e_{\tau}<550\right)$ and stratification levels and turbulence statistics at $R e_{\tau}=1000$ are documented, and the highest stratification level considered here is $R i_{\tau}=10^{4}$.

Fig. 5 shows the vertical profiles of mean variables and turbulence statistics for stably stratified open-channel flows at $R e_{\tau}=1000$. Here $\mathrm{S} 1\left(R i_{\tau}=100\right)$ and $\mathrm{S} 2\left(R i_{\tau}=10^{4}\right)$ correspond to the weakly and strongly stratified cases, respectively. As can be seen in Fig. 5a, with increasing the stratification level, the mean velocity variation is modified significantly. For S1, a relatively flat (well-mixed) velocity profile is observed in the channel, whereas for S2, the velocity accelerates drastically, especially near the top wall. The ratio of the velocities between the strongly stratified and the weakly stratified cases is approximately 4159 at the top channel, which is significantly larger than the ratio $(\sim 2)$ reported in Armenio 

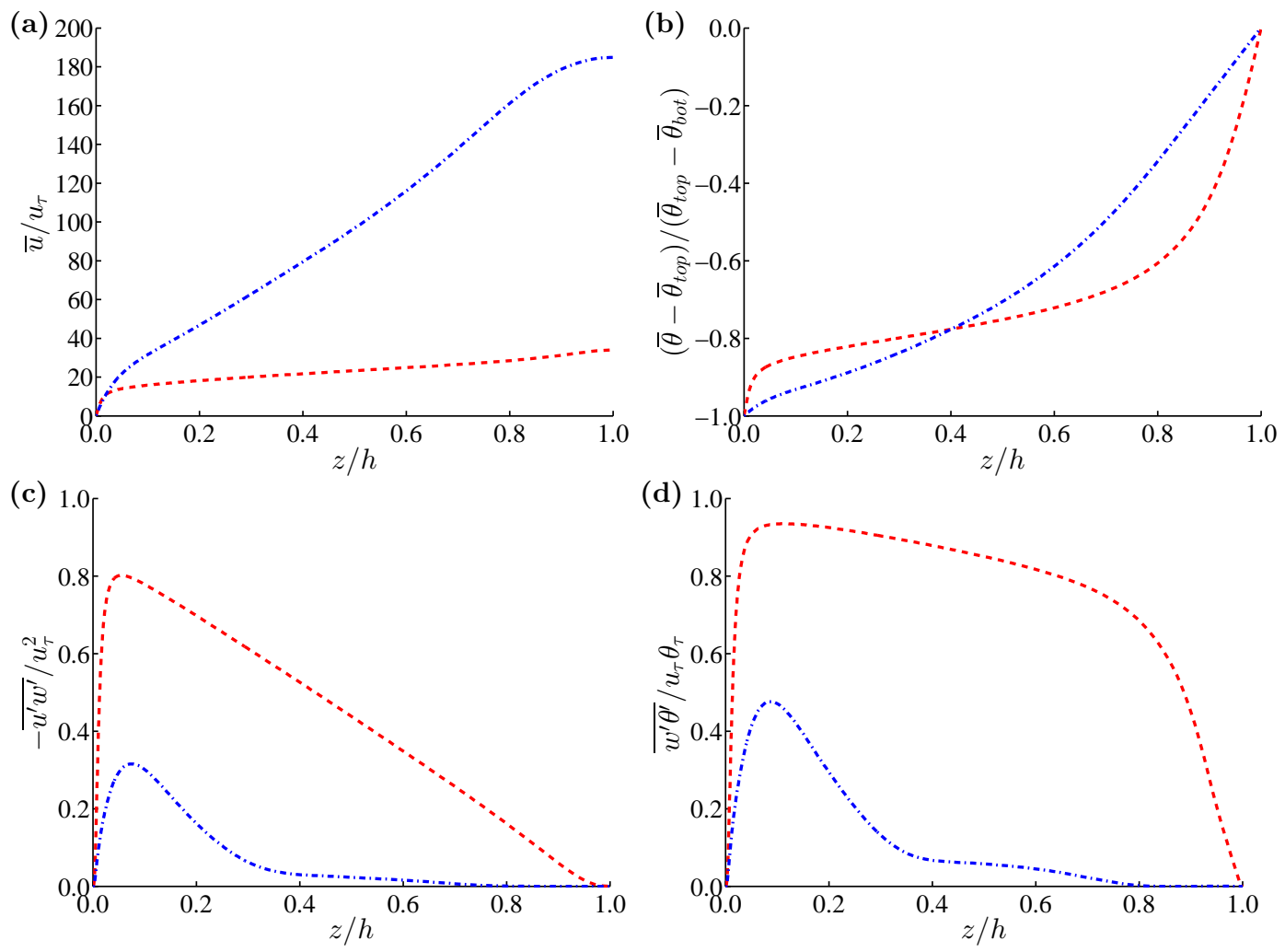

Figure 5: Vertical profiles of mean variables and turbulence statistics for stably stratified open-channel flows at $R e_{\tau}=1000$. - - : S1 $\left(R i_{\tau}=100\right) ;-\cdot-$ : S2 $\left(R i_{\tau}=10^{4}\right)$. (a) $\bar{u}$, (b) $\bar{\theta}$, (c) $\overline{u^{\prime} w^{\prime}}$, and (d) $\overline{w^{\prime} \theta^{\prime}}$.

and Sarkar [49] $\left(R e_{\tau}=180\right)$ and García-Villalba and del Álamo [17] $\left(R e_{\tau}=550\right)$. It is noted that the maximal amount of velocity acceleration can be obtained when $R i_{\tau}$ exceeds the laminarization threshold; a parabolic velocity profile is formed in this case. According to the linear stability theory in Gage and Reid [50], for $R e_{\tau}=1000$, the $R i_{\tau}$ threshold is $420 \sim 4.4 \times 10^{4}$. The current $R i_{\tau}$ value for $\mathrm{S} 2$ is still four times smaller than the laminarization threshold. This can be further confirmed by the fact that the maximal velocity for S2 is $u_{\max } / u_{\tau} \approx 185$, smaller than the maximal value from the parabolic profile at $R e_{\tau}=1000$, i.e., $u_{\max } / u_{\tau}=R e_{\tau} / 2=500$.

The acceleration of velocity is attributed to the drastic decrease of vertical momentum 425 flux (reduced mixing), as can be seen in Fig. 5c. The peak $-\overline{u^{\prime} w^{\prime}}$ decreases from $\sim 0.8$ (S1) to $\sim 0.35$ (S2). Moreover, the $-\overline{u^{\prime} w^{\prime}}$ value for $\mathrm{S} 2$ decreases substantially in $z / h>0.3$, implying a full laminarization of the flow. Quite interestingly, the current result indicates 
a coexistence of turbulent flows and fully laminarized flows, located in the lower and the upper part of the channel, respectively (see detailed illustration in Section 3.2). Note that in flux was also found to be significantly suppressed by the stratification. However, the decaying of turbulence was found to be transient and intermittent and no full (steady) laminarization was reported.

The mean temperature profile is also modified by the stratification as shown in Fig. 5b. stantial reduction of surface heat-flux as a result of the stable stratification. The Nusselt number $(N u)$ is a useful metric which reflects how close the flow is to a laminar state. $N u$ is defined as the ratio between heat transfer due to turbulence and pure conduction (laminar state): $N u=\frac{h}{\theta_{\text {top }}-\theta_{\mathrm{bot}}}\left(\frac{\partial \theta}{\partial z}\right)_{\mathrm{bot}}$. The $N u$ for $\mathrm{S} 1$ is 7.2 and it becomes 1.2 for S2, implying that the flow is becoming closer to laminar. Note that a linear mean temperature profile will be formed in a laminar state. Similar to those reported for the momentum flux, the turbulent heat flux is also found to be significantly suppressed by stratification, and a full laminarization is observed in the upper channel.

\subsection{Spatio-temporal characteristics of global intermittency}

The existence of global intermittency, or turbulent burst, in the very stable boundary layer is well-known in the literature. This intriguing phenomenon is typically characterized as an observed time-series alternating between quiescent (laminar) and bursty (turbulent) state. This type of intermittent time-series has been widely observed in various regions of the world $[51,52,53,54,55,56]$, to name a few. It is also documented in the literature that the globally intermittent turbulence portrays intriguing spatio-temporal correlations [57]. A number of studies have been conducted to investigate the generation and dynamics of global intermittency in the $\mathrm{ABL}[54,55,58,59,60]$, among others. In recent years, the global intermittency was successfully reproduced by a number of DNS studies [12, 17, 20, 21, 22], which complements the understanding of its spatio-temporal characteristics. In this section, we document the spatio-temporal variations of global intermittency at $R e_{\tau}=1000$. The similarity and discrepancy between the previously reported results and the current results are discussed.

A number of monitor points are placed in various vertical locations of the channel to document the time-variations of the stratified turbulent flows. Fig. 6 shows the time-series 

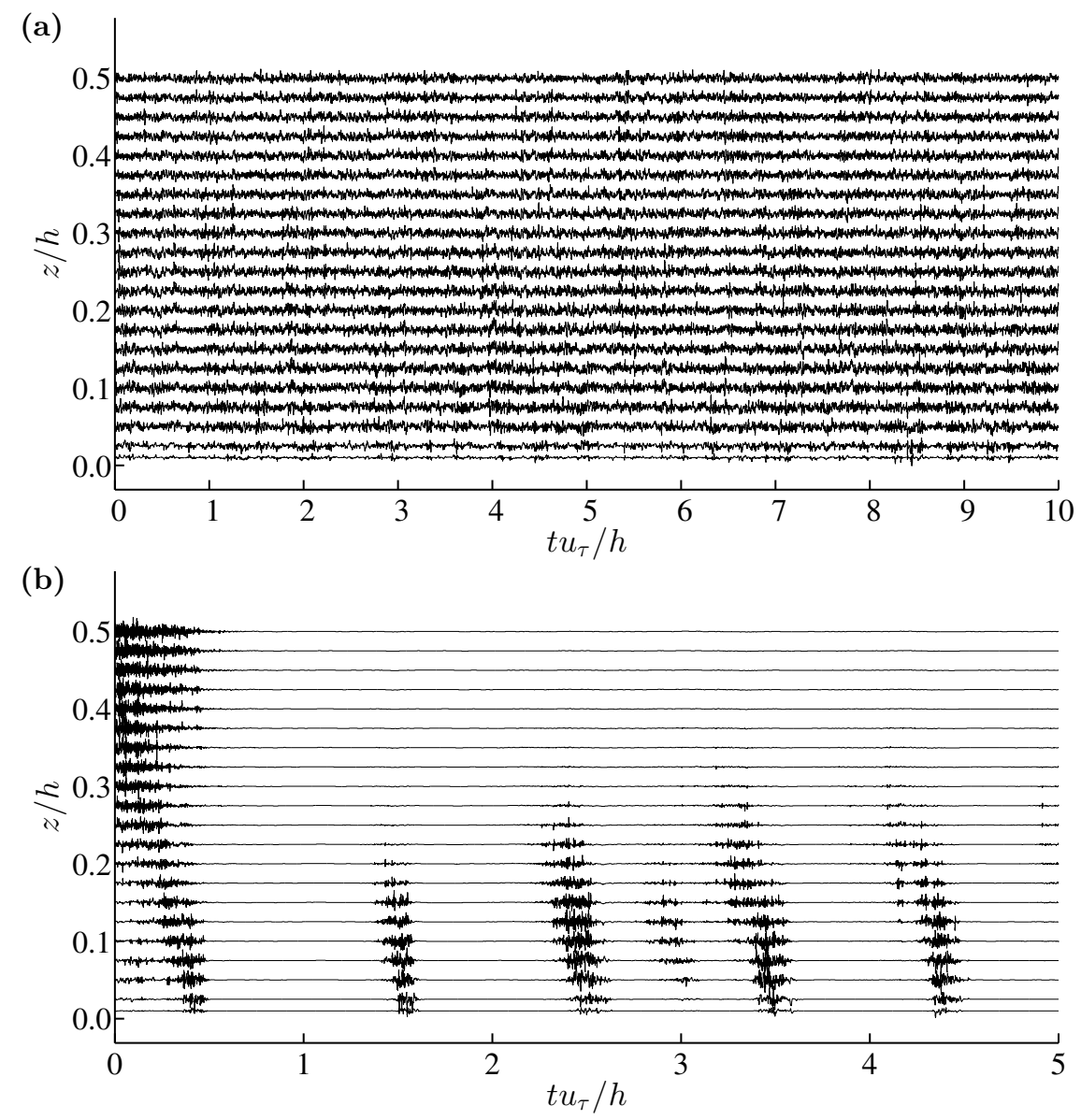

Figure 6: Time-series of vertical velocity at different locations in the channel. (a) $\mathrm{S} 1\left(R i_{\tau}=100\right)$, and (b) $\mathrm{S} 2\left(R i_{\tau}=10^{4}\right)$.

460 of vertical velocity at different locations in the channel. For S1, the turbulent flow is continuous and no intermittency is observed. However, for S2, alternating turbulent and laminar flows are clearly identified in the time-series, especially in the lower channel. In the vertical direction, the turbulent bursting event is triggered slightly earlier near the upper channel than the bottom wall. These features coincide with those reported in He and Basu [22] for low Re flows. However, in contrast to He and Basu [22] that the turbulence sustained in the upper channel, in the current case the turbulence is totally laminarized in $z / h>0.3$. Based on their DNS results, Ansorge and Mellado [21] showed that the turbulence laminarization is not an on-off process. Instead, the turbulence first becomes steadily intermittent and then fully laminarized with increasing the stratification level. This 
470 indicate that the full laminarization process is not synchronous in the vertical direction; this process first occurs in the upper channel due to the dominant buoyancy while the intermittent turbulence can still sustain in the lower channel at the same time.
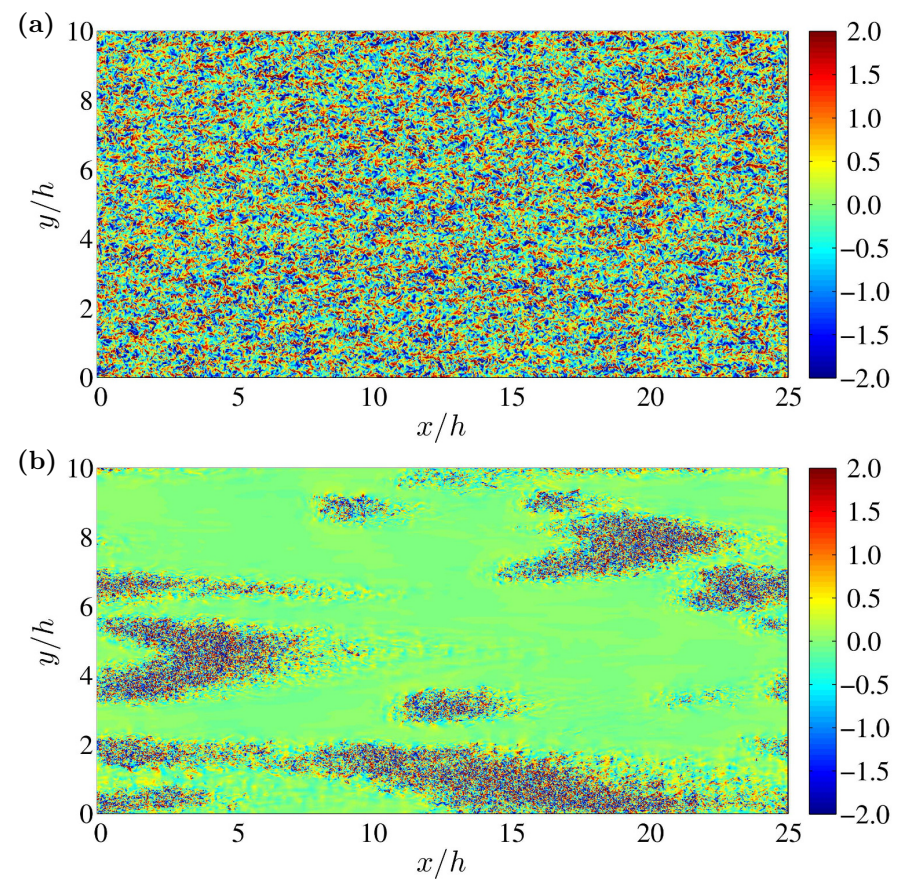

Figure 7: Contour of vertical velocity at the $x-y$ plane located at $z / h=0.1$. (a) $\mathrm{S} 1\left(R i_{\tau}=100\right)$, and (b) S2 $\left(R i_{\tau}=10^{4}\right)$.

According to Coulter and Doran [57], the intermittent time-series was found to be spa475 fully localized phenomenon. The spatial correlation of temporal intermittency was further corroborated by the DNS study in He and Basu [22]. In their study, the coexisting laminar-turbulent flows were found to be advecting with mainstream and triggering intermittent time-series signal. Investigation of the spatial pattern of coexisting 480 laminar-turbulent flows is important for the understanding of temporal intermittency. Furthermore, to what extent does the temporal intermittency spatially correlate with each other has implication for turbulence parameterization in mesoscale modeling [57].

Fig. 7 shows the contour of vertical velocity at the $x-y$ plane located at $z / h=0.1$. A horizontally homogeneous turbulent field is found for S1, whereas for S2, a coexistence of 

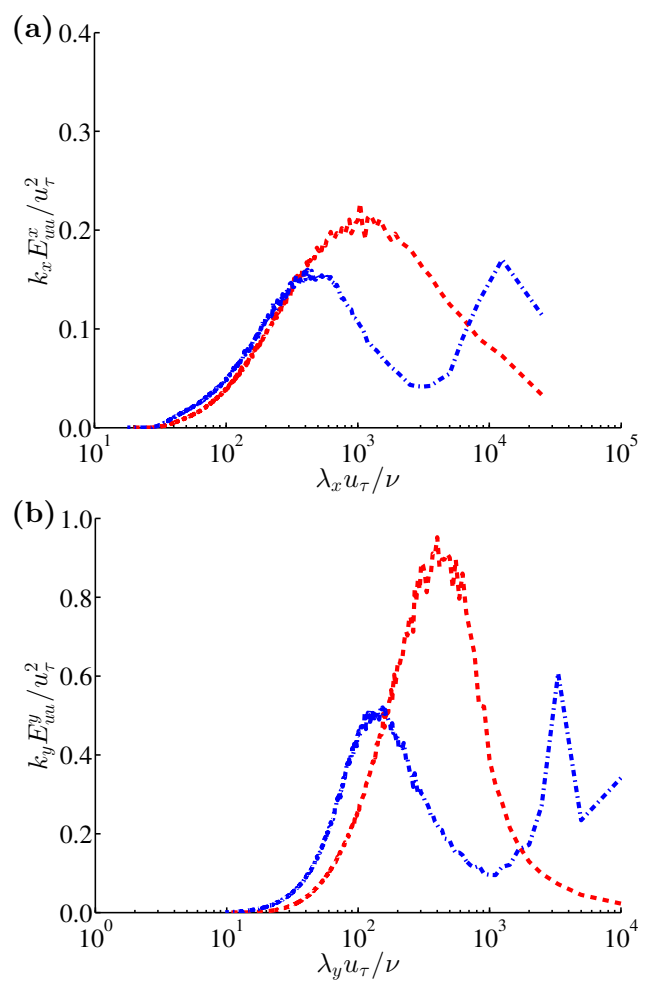

Figure 8: Premultiplied (a) streamwise and (b) spanwise spectra of $u$ at $z / h=0.1$. Here $E_{u u}^{x}$ and $E_{u u}^{y}$ are the 1D energy spectra of $u$ in the $x$ and $y$ directions, respectively. The corresponding wavenumbers are $k_{x}$ and $k_{y}$, and the corresponding wavelengths are $\lambda_{x}$ and $\lambda_{y} .--: \mathrm{S} 1\left(R i_{\tau}=100\right) ;-\cdot-: \mathrm{S} 2\left(R i_{\tau}=10^{4}\right)$.

laminar-turbulent bands is observed. Note that similar spatial intermittency was previously reported in DNS studies $[12,17,20,21,22]$ at relatively low Re. It would be interesting to evaluate if the spatial patterns of the coexisting laminar-turbulent flows have scaling features at a relatively high $R e$. For this purpose, we calculate the premultiplied streamwise and spanwise spectra of $u$ at $z / h=0.1$, as shown in Fig. 8. Here $E_{u u}^{x}$ and $E_{u u}^{y}$ are the 1D energy spectra of streamwise velocity in the $x$ and $y$ directions, respectively. The corresponding wavenumbers are $k_{x}$ and $k_{y}$, and the corresponding wavelengths are $\lambda_{x}$ and $\lambda_{y}$. Note that $\lambda_{x}$ and $\lambda_{y}$ are normalized in inner units, e.g., $\lambda_{x}^{+}=\lambda_{x} u_{\tau} / \nu$. The premultiplied spectra represent the contribution of streamwise velocity variance from an infinitesimal logarithmic wavelength interval at $\lambda_{x}$ or $\lambda_{y}$. One can see that, for the weakly stratified case 495 (S1), a single spectral peak is observed at $\lambda_{x}^{+} \approx 1000$ and $\lambda_{y}^{+} \approx 400$ for the streamwise and spanwise spectra, respectively. This peak corresponds to the alternating low- and high-speed 
streaks, i.e., the near-wall coherent structures, with characteristic lengths $\lambda_{x}^{+} \approx 1000$ and $\lambda_{y}^{+} \approx 400$. For the very stable case (S2), the characteristic lengths for the near-wall streaks decrease as a result of the suppression of buoyancy. This trend coincides well with that observed in the stably stratified closed-channel flows at $R e_{\tau}=550$ [17]. Moreover, for $\mathrm{S} 2$, another spectral peak is found at much larger characteristic wavelengths, i.e., $\lambda_{x}^{+} \approx 1.4 \times 10^{4}$ and $\lambda_{y}^{+} \approx 3.3 \times 10^{3}$. This large-scale peak is clearly associated with the spatial pattern of global intermittency. Note that in Brethouwer et al. [20], similar double-peak spectra were reported for strongly stratified open-channel flows. The corresponding wavelengths for intermittent pattern in the spanwise direction are $\lambda_{y}^{+} \approx 4.5 \times 10^{3}$ at $R e_{\tau}=192$ and $\lambda_{y}^{+} \approx 2.5 \times 10^{3}$ at $R e_{\tau}=334$. Although the current $R e_{\tau}$ is much larger than those reported in Brethouwer et al. [20], we do not observe clear dependence of intermittent pattern wavelengths on $R e_{\tau}$. It is suggested that the magnitude of intermittent pattern wavelength scales in inner units in $R e_{\tau}<1000$. This result implies that the pattern wavelengths decrease in outer units with increasing $R e_{\tau}$, i.e., $\lambda_{y} / h \approx 23.4,7.5$, and 3.3 for $R e_{\tau}=192,334$, and 1000 , respectively. It is important to note that the indication of the scaling behavior is only based on three $R e_{\tau}$, systematic evaluation of this topic at wider ranges of $R e_{\tau}$ is needed in the future work.

\subsection{Relation to Monin-Obukhov similarity theory}

The Monin-Obukhov similarity theory (MOST) is widely used to describe the fluxgradient relationships in the ABL. In the past decades, a number of similarity relationships for wind speed and temperature have been proposed based on observational data $[61,62,63,64]$, to name a few. In order to evaluate the relevance of the current DNS results to realistic atmospheric flow conditions, we compare the simulated flux-gradient similarity relationships with those observed in the ABL. According to the MOST, the normalized gradients of velocity and temperature can be related to the stability parameter $(\zeta)$ as:

$$
\begin{gathered}
\frac{\kappa z}{u_{*}} \frac{\partial \bar{u}}{\partial z}=\Phi_{m}(\zeta), \\
\frac{\kappa z}{\theta_{*}} \frac{\partial \bar{\theta}}{\partial z}=\Phi_{h}(\zeta) .
\end{gathered}
$$

Here $\kappa=0.4$ is the von Karman constant, $u_{*}=\left({\overline{u^{\prime} w^{\prime}}}^{2}+{\overline{v^{\prime} w^{\prime}}}^{2}\right)^{1 / 4}$ is the local friction velocity, $\theta_{*}=-\overline{w^{\prime} \theta^{\prime}} / u_{*}$ is the local temperature scale, and $\zeta=z / \Lambda$ with $\Lambda$ being the local Obukhov length defined as $\Lambda=-\bar{\theta} u_{*}^{3} / \kappa g \overline{w^{\prime} \theta^{\prime}}$. Fig. 9 shows the dependence of $\Phi_{m}$ and $\Phi_{h}$ 

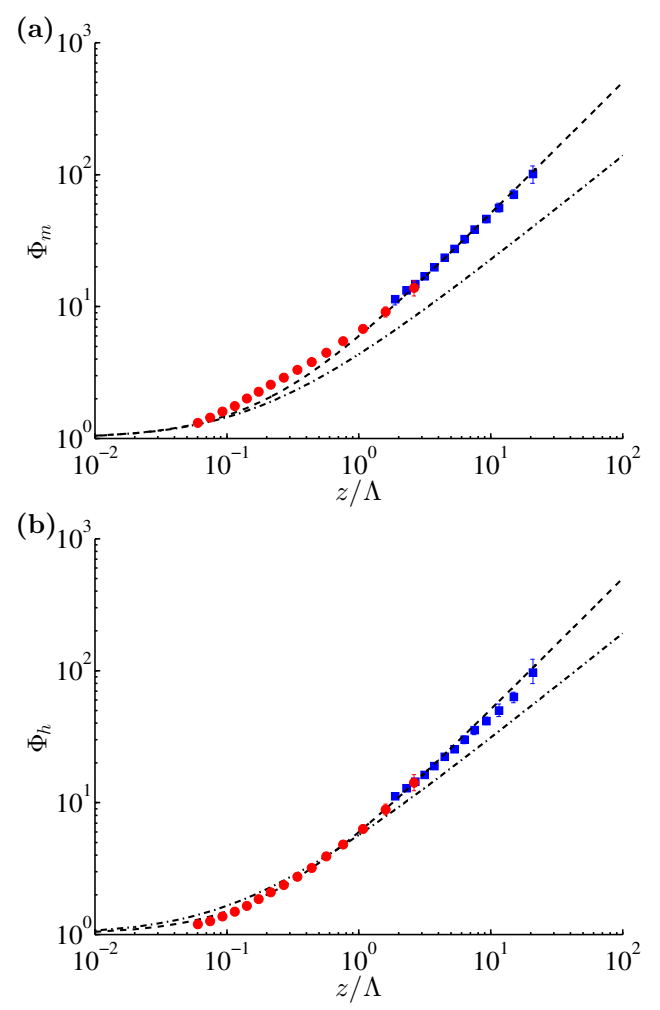

Figure 9: Dependence of (a) $\Phi_{m}$ and (b) $\Phi_{h}$ as a function of stability parameters. The symbols are the ensemble results constructed from the DNS data. ๑: S1 $\left(R i_{\tau}=100\right)$; $\mathbf{\square}$ : S2 $\left(R i_{\tau}=10^{4}\right)$. The lower and higher error bars represent 25 and 75 percentile value of the ensemble results, respectively. The lines are the similarity relationships proposed in Dyer [61] (--) and Duynkerke [62] (-・-).

as a function of the stability parameter. The symbols are the ensemble results based on the DNS data. For each simulation, the ensemble samples are based on the horizontal-plane averaged variables at different vertical locations and times (please refer to He and Basu [24] for more details of DNS-based similarity relationships). In order to avoid the impact of molecular viscosity at the bottom wall and the boundary condition at the top wall, the similarity relationships are constructed using the DNS data in $0.1<z / h<0.9$ for S1. ${ }_{530}$ For $\mathrm{S} 2$, an even shorter data range $(0.1<z / h<0.3)$ are used since the flows are fully laminarized in $z / h>0.3$. The lines are the existing similarity relationships, i.e., the linear functions: $\Phi_{m}=1+5 z / \Lambda, \Phi_{h}=1+5 z / \Lambda$ proposed in Dyer [61] (D74), and the nonlinear functions: $\Phi_{m}=1+5 z / \Lambda(1+6.25 z / \Lambda)^{-0.2}, \Phi_{h}=1+7.5 z / \Lambda(1+9.375 z / \Lambda)^{-0.2}$ proposed in Duynkerke [62] (D91). Although the weakly and strongly stratified cases (S1 and S2) 
cover different range of stability, they all agree reasonably well with those proposed based on the observational data, especially for D74. We do point out that there is a noticeable overestimation of $\Phi_{m}$ in the lower stability region $(0.1<z / \Lambda<0.6$ in Fig. 9a). Overall, the good agreement between the DNS-based similarity relationships and those proposed using observational data demonstrates the relevance of DNS results to atmospheric flow conditions. It is also noted that the stability parameter covers a much wider range, i.e., $(z / \Lambda)_{\max } \approx 20$, compared to the previous results using the low-Re DNS data, as reported in van de Wiel et al. [23], and He and Basu [24]. This feature highlights the advantage of using the current massively parallel DNS solver for simulating high-Re stably stratified flows.

\section{Conclusions}

In this paper, a high-order finite-difference direct numerical simulation (DNS) solver is developed for massively parallel simulations of incompressible stably stratified turbulent flows in open channels. The semi-implicit fractional time step method is used for time advancement, and the spatial derivatives are discretized using the fourth-order and second-order central differential schemes in the horizontal and vertical directions, respectively. The resulting discretization equations, including the momentum, temperature, and pressure equations, are then directly solved using the Fourier analysis approach. The 2D domain decomposition technique is utilized for parallel communication, and a reasonably good scalability is obtained with up to $32768 \mathrm{CPU}$ cores.

The newly developed DNS solver is then verified using the benchmark data in Moser et al. [37], and García-Villalba and del Álamo [17] for neutrally and stably stratified flows, and a reasonably good agreement is found. Moreover, two stably stratified open-channel simulations are conducted with a weak and a very strong stratification level. The bulk Reynolds number for the strongly stratified case is approximately $10^{5}$. The characteristics of strongly stratified flows are compared with those previously reported at relatively low Reynolds numbers, and several discrepancies are observed. For example, the acceleration of mean velocity is found to be much higher than the low Reynolds number cases. In addition, the laminarization process is found to be asynchronous in the vertical direction; this process first occurs in the upper channel due to the dominant buoyancy while the

intermittent turbulence can still sustain in the lower channel at the same time. In addition 
to the discrepancies, we observe intriguing scaling behavior for the spatial pattern of global intermittency. That is to say, the pattern wavelength is found to scale in inner units in $R e_{\tau}<$ 1000. In order to evaluate the relevance of the DNS-based results to the atmospheric flows, the DNS-based similar relationships are compared with those proposed using observational data, and a reasonably good agreement is found. It is also observed that the range of the stability parameter, i.e., $(z / \Lambda)_{\max } \approx 20$, from the current high-Re DNS-based similarity relationships is much wider compared with the existing low-Re DNS-based results, i.e., $(z / \Lambda)_{\max } \approx 5$.

Above all, the newly developed DNS solver is shown to be competent for massively parallel simulations of stably stratified channel flow at relatively higher Reynolds numbers. In the future, this solver will be used to generate a DNS dataset with a wide range of Reynolds numbers and Richardson numbers. Using this comprehensive dataset, various flux- and gradient-based similarity relationships will be constructed and compared with the existing similarity functions $[2,65,66]$ to further evaluate the relevance of DNS-based results. Moreover, new similarity relationships will be developed based on this DNS dataset, and systematic investigations will be conducted to document the turbulence characteristics in the "very stable" regime.

\section{Acknowledgements}

The author acknowledges financial support received from the Department of Defense (AFOSR grant under award number FA9550-12-1-0449). Any opinions, findings and conclusions or recommendations expressed in this material are those of the author and do not necessarily reflect the views of the Department of Defense. The author also acknowledges computational resources obtained from the U.S. Air Force Research Laboratory (AFRL), Army Research Laboratory (ARL), Department of Defense Supercomputing Resource Cen590 ter (DSRC), the Extreme Science and Engineering Discovery Environment (XSEDE), which is supported by National Science Foundation (grant number ACI-1053575). The author would like to thank Sukanta Basu and Adam DeMarco for their valuable comments and suggestions for improving the quality of the paper. In addition, the author thanks Adam DeMarco for his kind help on the DSRC systems. 


\section{Appendix A. Solver verification}

In this appendix, the solver verification is performed. Seven closed-channel simulations (both neurally and stably stratified; V1-V7 in Table 3) are conducted and the simulation results are compared with the benchmark data [17,37]. The boundary conditions and domain sizes are chosen to be identical to those used in Moser et al. [37], and GarcíaVillalba and del Álamo [17]. The grid resolutions are set to $\Delta x^{+} \leq 8.8$ and $\Delta y^{+} \leq 4.4$. The horizontal and vertical spatial-derivatives are discretized using the fourth order and second order central differential schemes, respectively, and a coupled Adams-Bashforth and Crank-Nicolson scheme is used for time advancement. The simulations are run for 20 nondimensional time (i.e., $t u_{\tau} / h=20$ ) for the calculation of turbulence statistics.

First, the simulation results produced by the current DNS solver are compared against the well-cited DNS benchmark (neutrally stratified case) from Moser et al. [37]. Fig. A.10 shows the vertical profiles of mean flows and turbulence statistics at $R e_{\tau}=180,395$, and 590. One can see that the results from the current solver agree well with those from Moser et al. [37]. We do point out that the momentum flux $\left(\overline{u^{\prime} w^{\prime}}\right)$ for $R e_{\tau}=395$ is underestimated at $z / h \approx 0.3$, see Fig. A.10d.

Next, the DNS results for the stably stratified flows are compared with the data (extracted by a digitizer) in García-Villalba and del Álamo [17]. Vertical profiles of mean flows and turbulence fluxes are shown in Fig. A.11. Again, the results from the current solver agree well with those from García-Villalba and del Álamo [17] for all the stratification levels. The maximal discrepancy is found for $\overline{w^{\prime} \theta^{\prime}}$ at $z / h \approx 1.0$.

Although some differences are observed between the results from the current DNS solver and those from Moser et al. [37], and García-Villalba and del Álamo [17], this level of discrepancy is not unexpected from different DNS solvers (please refer to the code verification in Kasagi et al., [67], Laizet and Lamballais [68], to name a few). In summary, the current solver accurately simulates the mean flows and turbulent statistics for the neutral and stratified cases. Without any doubt, these solver verification results build confidence for the simulations and analyses reported in the paper.

\section{References}

[1] R. B. Stull, An introduction to boundary layer meteorology, Vol. 13, Springer, 1988. 

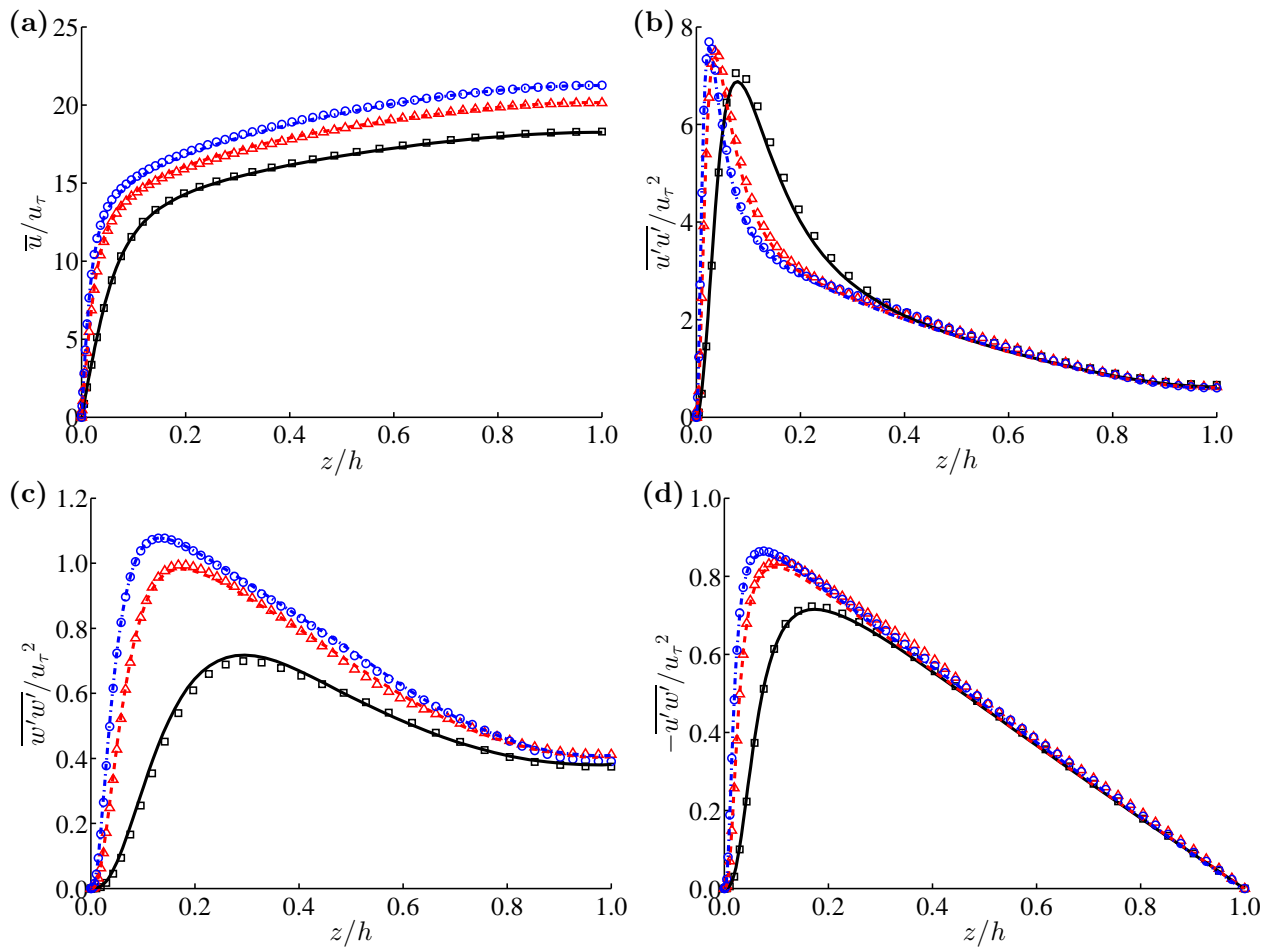

Figure A.10: Vertical profiles of mean variables and turbulence statistics for neutrally stratified closedchannel flows at $R e_{\tau}=180,395$, and 590. (a) $\bar{u}$, (b) $\overline{u^{\prime} u^{\prime}}$, (c) $\overline{w^{\prime} w^{\prime}}$, and (d) $\overline{u^{\prime} w^{\prime}}$. The lines are the simulation results from the current DNS solver. - $: R e_{\tau}=180$; - - $R e_{\tau}=395 ;-\cdot-$ : $R e_{\tau}=590$. The symbols are the benchmark data from Moser et al. [37]. $\square: R e_{\tau}=180 ; \triangle: R e_{\tau}=395 ; \bigcirc: R e_{\tau}=590$.

[2] Z. Sorbjan, Structure of the atmospheric boundary layer, Prentice Hall, 1989.

[3] J. R. Garratt, The Atmospheric Boundary Layer, Cambridge University Press, 1994.

[4] J. C. Wyngaard, Turbulence in the Atmosphere, Cambridge University Press, 2010.

[5] L. Mahrt, Stably stratified atmospheric boundary layers, Annual Review of Fluid Mechanics 46 (2014) 23-45.

[6] B. Storm, J. Dudhia, S. Basu, A. Swift, I. Giammanco, Evaluation of the weather research and forecasting model on forecasting low-level jets: Implications for wind energy, Wind Energy 12 (1) (2008) 81-90.

[7] C. G. Nunalee, S. Basu, Mesoscale modeling of coastal low-level jets: implications for offshore wind resource estimation, Wind Energy 17 (2013) 1199-1216. 

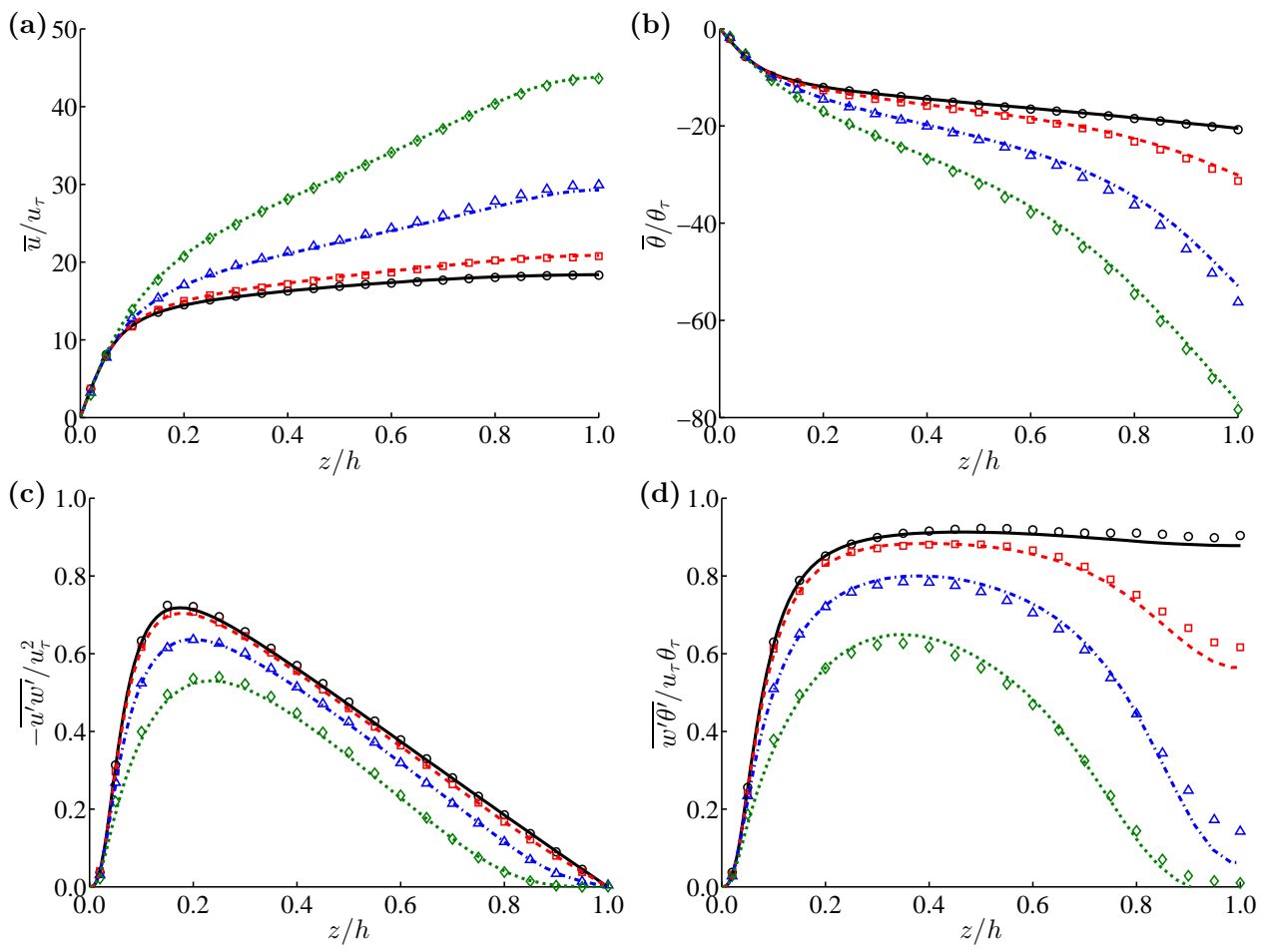

Figure A.11: Vertical profiles of mean variables and turbulence statistics for stably stratified closed-channel flows at $R e_{\tau}=180$. (a) $\bar{u}$, (b) $\bar{\theta}$, (c) $\overline{u^{\prime} w^{\prime}}$, and (d) $\overline{w^{\prime} \theta^{\prime}}$. The lines are the simulation results from the current DNS solver. - $: R i_{\tau}=0 ;--: R i_{\tau}=18 ;-\cdot-: R i_{\tau}=120 ; \cdots: R i_{\tau}=480$. The symbols are the benchmark data from García-Villalba and del Álamo [17]. $\bigcirc: R i_{\tau}=0 ; \square: R i_{\tau}=18 ; \triangle: R i_{\tau}=240 ; \diamond: R i_{\tau}=480$.

[8] J. A. Salmond, I. G. McKendry, A review of turbulence in the very stable nocturnal boundary layer and its implications for air quality, Progress in Physical Geography 29 (2) (2005) 171-188.

[9] V. Armenio, S. Sarkar, Environmental Stratified Flows, Springer, 2005.

[10] H. J. S. Fernando, Handbook of Environmental Fluid Dynamics: Overview and Fundamentals, CRC Press, 2012.

[11] R. A. Pielke, R. P. Pearce, Mesoscale modeling of the atmosphere, Vol. 25, Springer, 1994.

[12] O. Flores, J. J. Riley, Analysis of turbulence collapse in the stably stratified surface layer using direct numerical simulation, Boundary-Layer Meteorology 139 (2) (2011) $241-259$. 
[13] F. T. M. Nieuwstadt, Direct numerical simulation of stable channel flow at large stability, Boundary-Layer Meteorology 116 (2) (2005) 277-299.

[14] J. M. M. Donda, I. G. S. van Hooijdonk, A. F. Moene, H. J. J. Jonker, G. J. F. van Heijst, H. J. H. Clercx, B. J. H. van de Wiel, Collapse of turbulence in stably stratified channel flow: a transient phenomenon, Quarterly Journal of the Royal Meteorological Society 141 (2015) 2137-2147.

[15] O. Iida, N. Kasagi, Y. Nagano, Direct numerical simulation of turbulent channel flow under stable density stratification, International Journal of Heat and Mass Transfer 45 (8) (2002) 1693-1703.

[16] R. P. Garg, J. H. Ferziger, S. G. Monismith, J. R. Koseff, Stably stratified turbulent channel flows. I. stratification regimes and turbulence suppression mechanism, Physics of Fluids 12 (2000) 2569-2594.

[17] M. García-Villalba, J. C. del Álamo, Turbulence modification by stable stratification in channel flow, Physics of Fluids 23 (2011) p. 045104.

[18] S. K. Shah, E. Bou-Zeid, Direct numerical simulations of turbulent ekman layers with increasing static stability: modifications to the bulk structure and second-order statistics, Journal of Fluid Mechanics 760 (2014) 494-539.

[19] J. C. Barnard, Intermittent turbulence in the very stable Ekman layer, Ph.D. thesis (2000).

[20] G. Brethouwer, Y. Duguet, P. Schlatter, Turbulent-laminar coexistence in wall flows with Coriolis, buoyancy or Lorentz forces, Journal of Fluid Mechanics 704 (2012) 137172.

[21] C. Ansorge, J. P. Mellado, Global intermittency and collapsing turbulence in the stratified planetary boundary layer, Boundary-Layer Meteorology (2014) 1-28.

$670[22]$ P. He, S. Basu, Direct numerical simulation of intermittent turbulence under stably stratified conditions, Nonlinear Processes in Geophysics 22 (2015) 447-471.

[23] B. J. H. van de Wiel, A. F. Moene, W. H. de Ronde, H. J. J. Jonker, Local similarity in the stable boundary layer and mixing-length approaches: consistency of concepts, Boundary-Layer Meteorology 128 (1) (2008) 103-116. 
[24] P. He, S. Basu, Development of similarity relationships for energy dissipation rate and temperature structure parameter in stably stratified flows: A direct numerical simulation approach, Environmental Fluid Mechanicsdoi : 10.1007/s10652-015-9427-y.

[25] A. A. Townsend, The structure of turbulent shear flow, Cambridge university press, 1980.

[26] Z. Sorbjan, A. A. Grachev, An evaluation of the flux-gradient relationship in the stable boundary layer, Boundary-Layer Meteorology 135 (3) (2010) 385-405.

[27] C. Canuto, M. Y. Hussaini, A. Quarteroni, T. A. Zang, Spectral Methods in Fluid Dynamics, Springer, 1988.

[28] P. Moin, K. Mahesh, Direct numerical simulation: a tool in turbulence research, Annual Review of Fluid Mechanics 30 (1) (1998) 539-578.

[29] Y. Zang, R. L. Street, J. R. Koseff, A non-staggered grid, fractional step method for time-dependent incompressible navier-stokes equations in curvilinear coordinates, Journal of Computational Physics 114 (1) (1994) 18-33.

[30] A. J. Chorin, Numerical solution of the Navier-Stokes equations, Mathematics of Computation 22 (104) (1968) 745-762.

[31] J. Kim, P. Moin, Application of a fractional-step method to incompressible NavierStokes equations, Journal of Computational Physics 59 (2) (1985) 308-323.

[32] S. Laizet, N. Li, Incompact3d: A powerful tool to tackle turbulence problems with up to $\mathrm{o}\left(10^{5}\right)$ computational cores, International Journal for Numerical Methods in Fluids 67 (11) (2011) 1735-1757.

[33] A. W. Vreman, J. G. M. Kuerten, Comparison of direct numerical simulation databases of turbulent channel flow at $R e_{\tau}=180$, Physics of Fluids 26 (2014) p. 015102.

[34] Top500 Lists, Top500 lists november 2014.

[35] S. Laizet, J. C. Vassilicos, Direct numerical simulation of fractal-generated turbulence, in: Direct and Large-Eddy Simulation VII, Springer, 2010, pp. 17-23.

[36] M. Lee, R. D. Moser, Direct numerical simulation of turbulent channel flow up to $R e_{\tau}=5200$, Journal of Fluid Mechanics (under review). 
[37] R. D. Moser, J. Kim, N. N. Mansour, Direct numerical simulation of turbulent channel flow up to $R e_{\tau}=590$, Physics of Fluids 11 (4) (1999) 943-945.

[38] D. Rosenberg, A. Pouquet, R. Marino, P. D. Mininni, Evidence for Bolgiano-Obukhov scaling in rotating stratified turbulence using high-resolution direct numerical simulations, Physics of Fluids 27 (5) (2015) 055105.

[39] S. M. de Bruyn Kops, Classical scaling and intermittency in strongly stratified Boussinesq turbulence, Journal of Fluid Mechanics 775 (2015) 436-463.

[40] P. Orlandi, Fluid flow phenomena: a numerical toolkit, Springer Science \& Business Media, 2000.

[41] F. H. Harlow, J. E. Welch, Numerical calculation of time-dependent viscous incompressible flow of fluid with free surface, Physics of Fluids 8 (12) (1965) 2182-2189.

[42] N. A. Kampanis, J. A. Ekaterinaris, A staggered grid, high-order accurate method for the incompressible navier-stokes equations, Journal of Computational Physics 215 (2) (2006) 589-613.

[43] Y. Morinishi, T. S. Lund, O. V. Vasilyev, P. Moin, Fully conservative higher order finite difference schemes for incompressible flow, Journal of Computational Physics 143 (1) (1998) 90-124.

[44] K. Horiuti, T. Itami, Truncation error analysis of the rotational form for the convective terms in the navier-stokes equation, Journal of Computational Physics 145 (2) (1998) $671-692$.

[45] D. Pekurovsky, P3dfft: A framework for parallel computations of Fourier transforms in three dimensions, SIAM Journal on Scientific Computing 34 (4) (2012) C192-C209.

[46] N. Li, S. Laizet, 2decomp\&fft-a highly scalable $2 \mathrm{~d}$ decomposition library and fft interface, in: Cray User Group 2010 conference, Edinburgh, 2010.

[47] FFTW, Fftw documentation.

URL http://www.fftw.org/fftw3_doc/ 
[48] M. Lee, N. Malaya, R. D. Moser, Petascale direct numerical simulation of turbulent channel flow on up to 786k cores, in: Proceedings of the International Conference on High Performance Computing, Networking, Storage and Analysis, ACM, 2013, p. 61.

[49] V. Armenio, S. Sarkar, An investigation of stably stratified turbulent channel flow using large-eddy simulation, Journal of Fluid Mechanics 459 (2002) 1-42.

[50] K. S. Gage, W. H. Reid, The stability of thermally stratified plane Poiseuille flow, Journal of Fluid Mechanics 33 (1968) 21-32.

[51] R. Nakamura, L. Mahrt, A study of intermittent turbulence with CASES-99 tower measurements, Boundary-Layer Meteorology 114 (2) (2005) 367-387.

[52] B. Holtslag, Preface: GEWEX atmospheric boundary-layer study (GABLS) on stable boundary layers, Boundary-Layer Meteorology 118 (2) (2006) 243-246.

[53] N. J. Cullen, K. Steffen, P. D. Blanken, Nonstationarity of turbulent heat fluxes at Summit, Greenland, Boundary-Layer Meteorology 122 (2) (2007) 439-455.

[54] J. Sun, S. P. Burns, D. H. Lenschow, R. Banta, R. Newsom, R. Coulter, S. Frasier, T. Ince, C. Nappo, J. Cuxart, W. Blumen, X. Lee, X.-Z. Hu, Intermittent turbulence associated with a density current passage in the stable boundary layer, Boundary-Layer Meteorology 105 (2) (2002) 199-219.

[55] J. Sun, D. H. Lenschow, S. P. Burns, R. M. Banta, R. K. Newsom, R. Coulter, S. Frasier, T. Ince, C. Nappo, B. B. Balsley, M. Jensen, L. Mahrt, D. Miller, B. Skelly, Atmospheric disturbances that generate intermittent turbulence in nocturnal boundary layers, Boundary-Layer Meteorology 110 (2) (2004) 255-279.

[56] J. Sun, L. Mahrt, R. M. Banta, Y. L. Pichugina, Turbulence regimes and turbulence intermittency in the stable boundary layer during CASES-99, Journal of the Atmospheric Sciences 69 (1) (2012) 338-351.

[57] R. L. Coulter, J. C. Doran, Spatial and temporal occurrences of intermittent turbulence during CASES-99, Boundary-Layer Meteorology 105 (2) (2002) 329-349.

[58] B. J. H. van de Wiel, R. J. Ronda, A. F. Moene, H. A. R. de Bruin, A. A. M. Holtslag, Intermittent turbulence and oscillations in the stable boundary layer over land. part i: A bulk model, Journal of the Atmospheric Sciences 59 (5) (2002) 942-958. 
[59] B. J. H. van de Wiel, A. F. Moene, R. J. Ronda, H. A. R. de Bruin, A. A. M. Holtslag, Intermittent turbulence and oscillations in the stable boundary layer over land. part ii: A system dynamics approach, Journal of the Atmospheric Sciences 59 (17) (2002) $2567-2581$.

[60] B. J. H. van de Wiel, A. F. Moene, O. K. Hartogensis, H. A. R. de Bruin, A. A. M. Holtslag, Intermittent turbulence in the stable boundary layer over land. part iii: A classification for observations during CASES-99, Journal of the Atmospheric Sciences 60 (20) (2003) 2509-2522.

[61] A. Dyer, A review of flux-profile relationships, Boundary-Layer Meteorology 7 (3) (1974) 363-372.

[62] P. G. Duynkerke, Radiation fog: A comparison of model simulation with detailed observations, Monthly Weather Review 119 (2) (1991) 324-341.

[63] J. A. Businger, J. C. Wyngaard, Y. Izumi, E. F. Bradley, Flux-profile relationships in the atmospheric surface layer, Journal of the Atmospheric Sciences 28 (2) (1971) $181-189$.

[64] A. Beljaars, A. Holtslag, Flux parameterization over land surfaces for atmospheric models, Journal of Applied Meteorology 30 (3) (1991) 327-341.

[65] F. T. Nieuwstadt, The turbulent structure of the stable, nocturnal boundary layer, Journal of the Atmospheric Sciences 41 (14) (1984) 2202-2216.

[66] Z. Sorbjan, Gradient-based scales and similarity laws in the stable boundary layer, Quarterly Journal of the Royal Meteorological Society 136 (650) (2010) 1243-1254.

[67] N. Kasagi, Y. Tomita, A. Kuroda, Direct numerical simulation of passive scalar field in a turbulent channel flow, Journal of Heat Transfer 114 (1992) 598-606.

[68] S. Laizet, E. Lamballais, High-order compact schemes for incompressible flows: A simple and efficient method with quasi-spectral accuracy, Journal of Computational Physics 228 (16) (2009) 5989-6015. 